\title{
Discretised Link Travel Time Models based on Cumulative Flows: Formulations and Properties
}

\author{
Jiancheng Long * \\ School of Economics and Management, Beijing University of Aeronautics and Astronautics, \\ Beijing 100191, China \\ Ziyou Gao ${ }^{\dagger}$ \\ School of Traffic and Transportation, Beijing Jiaotong University, Beijing 100044, China \\ W.Y. Szeto $\ddagger$ \\ Department of Civil Engineering, The University of Hong Kong, Pokfulam Road, Hong Kong, China
}

\begin{abstract}
In the research area of dynamic traffic assignment, link travel times can be derived from link cumulative inflow and outflow curves which are generated by dynamic network loading. In this paper, the profiles of cumulative flows are piecewise linearized. Both the step function (SF) and linear interpolation (LI) are used to approximate cumulative flows over time. New formulations of the SF-type and LI-type link travel-time models are developed. We prove that these two types of link travel time models ensure first-in-first-out (FIFO) and continuity of travel time with respect to flows, and have other desirable properties. Since the LI-type link travel time model does not satisfy the causality property, a modified LI-type (MLI-type) link travel time model is proposed in this paper. We prove that the MLI-type link travel time model ensures causality, strong FIFO and travel time continuity, and that the MLI-type link travel time function is strictly monotone under the condition that the travel time of each vehicle on a link is greater than the free flow travel time on that link. Numerical examples are set up to illustrate the properties and accuracy of the three models.
\end{abstract}

Keywords: Link travel time; cumulative flow; piecewise linearization; FIFO; causality

\section{Introduction}

The properties of dynamic traffic assignment (DTA) have important implications on its ability to portray the actual travel behavior and computation speed. These properties depend strongly on two components

\footnotetext{
*Tel.: +86 1082316608, E-mail address: jianchenglong@buaa.edu.cn

†Tel.: +86 105168 8193, E-mail address: gaoziyou@jtys.bjtu.edu.cn

†Tel.: +852-28578552, E-mail address: ceszeto@hku.hk
} 
of DTA (Szeto and Lo, 2006): the travel choice principle and the traffic flow component.

The travel choice principle in DTA models travelers' propensity to travel, e.g., how they select their routes, departure times, modes, or destinations. In making such choices, travel time is one important element of their considerations. The dynamic user optimal (DUO) principle is in general adopted as the travel choice principle in DTA, which assumes that travelers select their routes and/or departure times to minimize their actual travel costs such as travel times. The travel choice principle can be mathematically formulated as a variational inequality problem (e.g., Friesz et al., 1993; Ran and Boyce, 1996; Lo and Szeto, 2002; Huang and Lam, 2002), where link travel times are functions of link flows. The existence of solutions to this problem requires the mapping function of the problem to be continuous and the solution set to be a nonempty compact convex set while the uniqueness of the solution further requires the mapping function to be strictly monotonic (Nagurney, 1993). These requirements imply that link travel times must be continuous with respect to link flows for solution existence; moreover, link travel times must be strictly monotone with respect to link flows for solution uniqueness. If the continuity cannot be guaranteed, the DTA problem can have no solution and the solution algorithms cannot give a convergent solution. Furthermore, if the travel time function is monotone, DTA can be solved by some existing solution methods efficiently. Therefore, continuity and monotonicity of travel times are two important properties in DTA.

The traffic flow component depicts how traffic propagates inside a traffic network and hence governs the network performance in terms of travel time. The procedure in implementation is often called dynamic network loading (DNL). In developing such component, one approach is to develop link travel time models. Link travel time models often express the travel time of a link as a function of the flow on that link. The link travel time models presented in the literature generally focus on the following properties: first-in firstout (FIFO) (e.g., Astarita, 1996; Huang and Lam, 2002; Carey et al., 2003; Carey and Ge, 2005; Carey and Ge, 2007), causality (e.g., Friesz et al., 1993; Astarita, 1996; Carey et al., 2003; Carey and Ge, 2007), and reduction to a static model (e.g., Carey et al., 2003; Carey and Ge, 2007). FIFO implies that vehicles that enter the link earlier will leave it sooner. Causality means that the speed and travel time of a vehicle on a link is only affected by the speed of vehicles ahead. Reduction to a static model means the link travel time function should reduce to the well-known static version when traffic flows are constant over time. Indeed, FIFO and causality are two actual traffic behaviors. A dynamic link travel time model is necessary to satisfy FIFO and causality in order to obtain the solutions of DTA that are consistent with actual traffic behavior.

The second approach to develop the travel flow component is based on either exit flow functions (e.g., Merchant and Nemhauser, 1978; Carey and Srinivasan, 1993; Lam and Huang, 1995; Wie and Tobin, 1998; Shin et al., 2000) or advanced exit flow functions (e.g., Kuwahara and Akamatsu, 2001; Lo and Szeto, 2002; Lian et al., 2007; Yperman, 2007; Szeto, 2008; Nie and Zhang, 2010). The exit flow function approach treats the outflow of a link or a segment of link as a non-decreasing function of the 
number of vehicles on the whole link or the link segment respectively. The advanced exit flow functions are developed based on either Daganzo's (1994, 1995a) solution scheme (referred to as the cell transmission model (CTM)) or Newell's (1993) solution scheme to the Lighthill and Whitham (1955) and Richards (1956) (LWR) hydrodynamic model of traffic flow. The main difference between these functions and the exit flow functions is that the advanced exit flow functions consider storage capacity to capture the effects of physical queues like queue spillback. If link inflow rates are given, the cumulative outflows can be generated by a DNL model. No matter whether the exit flow functions or the advanced exit flow functions are used for the DNL model, after the outflow and inflow rates of each link are determined, the cumulative flows and hence travel times can be obtained.

Travel times derived from cumulative flow curves is a fundamental step in the algorithms of many DTA models, and hence it is important to develop accurate and efficient method to do this. For example, the differences between policy scenarios studied using DTA models are often small. Hence it is important that other approximation or discretization errors must be kept even smaller relative to those. Indeed, discretization of time is needed to obtain travel time and even solve continuous time DTA models, since there are no known methods for solving complex continuous time models analytically. The outcome is that even if the cumulative curves have desirable properties (e.g, FIFO, causality, etc), the fineness of time discretization affects whether the discretised model (and in particular the travel time functions) retain these desirable properties. One may use a fine discretization to try to retain these properties so that the DNL is consistent with trave time estimation and that the overall model is theoretically sound. Nevertheless, our experience, and that of many authors worked with many DTA models and algorithms are that it is not computationally tractable to use very fine discretisations, as it can take excessive amounts of time even for medium or small networks. It is therefore important to investigate (i) whether the discretised travel time functions derived from cumulative flow curves with desirable properties can retain these properties, and (ii) how this is affected by the fineness of time discretisation. However, in the literature, travel times based on cumulative flows are calculated according to their proposed methods, and there are no travel time formulations derived from these flows for analysis of the properties of travel time.

This paper develops three link travel time formulations for DTA based on cumulative flows, which are different from traditional link travel time models that formulate travel time as a function of time-varying link flows (e.g., Daganzo, 1995b; Carey et al., 2003; Carey and Ge, 2007). Two of the three formulations are reformulated from existing travel time models for analysis purposes and the remaining formulation is new. Each cumulative flow profile is approximated by either a step function (SF) or linear interpolation (LI). In particular, the first formulation, namely the SF-type formulation, approximates the profiles of link cumulative inflows and outflows by step functions whereas the other two formulations, namely, the LI-type and the proposed modified LI-type (MLI-type) formulations, approximate the profiles by linear interpolations. The proposed formulations allow us to analyze the properties of the corresponding travel 
time functions including continuity, monotonicity, FIFO, and causality. Moreover, the accuracy of travel times derived from cumulative flow curves is unknown until this study using a numerical method.

This paper also discusses the properties of the three novel formulations. Note that the property of reduction to a static model is not considered in this paper but will be left for future research. For clarity, we restate the definitions of FIFO and causality discussed by Carey et al. (2003) here:

Property 1 (FIFO). This can be stated in various equivalent ways, for example, traffic that enters a link up to any time $t$ will exit from the link before traffic that enters after time $t$. This is not intended to preclude individual vehicles, traveling in the same direction, overtaking and passing each other. Hence, for traffic entering at say time $t$, the exit time can be interpreted as an 'average' exit time, or the exit time for an 'average' vehicle.

Property 2 (Causality). The link travel times (and hence outflow rates) for traffic entering at time $t$ depend on the traffic entering at time $t$ and earlier than $t$, but not on traffic entering later than $t$. This is sometimes referred to as a causality property.

The paper is organized as follows: in the next section, the general concept of link travel time derived by cumulative flows is presented in both continuous and discretized time settings. Section 3 presents the three novel formulations, and their properties are further investigated. Numerical experiments are given in Section 4 to illustrate the properties of the three travel time models and how the results are affected by the fineness of the discretization. Finally, Section 5 concludes this paper.

\section{Cumulative flows and link travel time}

\subsection{Continuous link travel time model}

Through this paper, we assume that the cumulative inflows and outflows are given. Equivalently, we assume that the cumulative inflows are given and the cumulative outflows are obtained from cumulative inflows by any DNL models. The cumulative number of vehicles that enter (exit) link $a$ at time $t$ is indicated as $U_{a}(t)\left(V_{a}(t)\right)$. The profiles of cumulative flows have the following two properties:

Property 3. The cumulative inflow $U_{a}(t)$ and cumulative outflow $V_{a}(t)$ are monotonically non-decreasing functions with respect to time $t$. That is, $U_{a}\left(t_{2}\right) \geq U_{a}\left(t_{1}\right)$ if $t_{2}>t_{1}$.

Property 4. The cumulative inflow $U_{a}(t)$ is not less than the cumulative outflow $V_{a}\left(t+\tau_{a}^{0}\right)$, where $\tau_{a}^{0}$ denotes the free flow travel time on link $a$. That is, $U_{a}(t) \geq V_{a}\left(t+\tau_{a}^{0}\right)$.

Monotonicity is a basic characteristic of cumulative flow functions. Property 4 implies that vehicles' travel times on a link cannot be less than the free flow travel time on the link.

As shown in Fig. 1, link travel time is related to the cumulative flows with the relationship $U_{a}(t)=$ $V_{a}\left(t+\tau_{a}(t)\right)$, where $\tau_{a}(t)$ is the travel time of link $a$ with respect to time $t$. If $U_{a}(t)$ and $V_{a}(t)$ are strictly increasing with respect to $t$, then the dynamic link travel times can be formulated as follows:

$$
\tau_{a}(t)=V_{a}^{-1}\left(U_{a}(t)\right)-t
$$


where $V_{a}^{-1}(\cdot)$ is the inverse function of $V_{a}(\cdot)$. Note that in deriving Eq. (1), FIFO is assumed to hold.

However, cumulative flow curves cannot always be strictly increasing due to two major factors (Nie, 2003):(1) a temporary inflow drop to zero (e.g., during light traffic), and (2) a temporary outflow capacity drop to zero (e.g., during a red phase, an incident or an accident). In these cases, Eq. (1) cannot be used. Nie (2003) developed a generic approach to retrieve link travel times from DNL results, in which the curves are only required to be non-decreasing and the method of linear interpolation is used to deal with the special case that the curves are non-decreasing for some time.

This paper provides another approach to compute the travel time $\tau_{a}(t)$ when the cumulative inflows and outflows are non-decreasing. This approach relies on a pretreatment of the link inflow rate $u_{a}(t)$ and the outflow capacity $s_{a}(t)$ before using Eq. (1) to compute $\tau_{a}(t)$. Let $\varepsilon$ and $\zeta$ be two very small positive numbers, where $\zeta$ should be far less than $\varepsilon$. Then, we set the inflow rate and the maximum outflow rate as $\max \left\{u_{a}(t), \varepsilon\right\}$ and $\max \left\{s_{a}(t), \zeta\right\}$ during the DNL implementation, respectively. This modification can ensure $u_{a}(t)>0$ and $s_{a}(t)>0$. Assuming that a small change in the inflow pattern will incur a small change in the outflow pattern. Then, if $\varepsilon$ is small enough (i.e., $\varepsilon \rightarrow 0^{+}$), the link travel times will not be changed much. Similarly, if $\zeta$ is small enough (i.e., $\zeta \ll \varepsilon$ and $\zeta \rightarrow 0^{+}$), the link travel times will not be changed much. Hence, with the proposed pretreatment, we have $u_{a}(t)>0$ and $s_{a}(t)>0$ for all time instants, and $U_{a}(t)$ and $V_{a}(t)$ are strictly increasing with respect to time $t$. Eq. (1) can then be used to calculate $\tau_{a}(t)$ approximately.

Definition 1 (Link FIFO of road traffic flow). The condition that the road traffic flow satisfies link FIFO is equivalent to the condition that the actual link travel time $\tau_{a}(t)$ satisfies link FIFO. This condition is satisfied if and only if

$$
t^{\prime}>t^{\prime \prime} \Rightarrow t^{\prime}+\tau_{a}\left(t^{\prime}\right) \geq t^{\prime \prime}+\tau_{a}\left(t^{\prime \prime}\right)
$$

In other words, the link FIFO condition of road traffic flow is satisfied if vehicles that enter the link earlier will leave sooner.

Definition 2 (Causality of road traffic flow) The condition that the road traffic flow satisfies causality is equivalent to the condition that the actual link travel time $\tau_{a}(t)$ satisfies causality. This condition is satisfied if $\tau_{a}(t)$ depends on the traffic entering at time $t$ and earlier than $t$, but not on traffic entering later than $t$.

Whether road traffic flow satisfies link FIFO and causality is mainly determined by which type of DNL model is used. In this paper, we assume that the road traffic flow both satisfies link FIFO and causality, or the link travel time calculated by Eq. (1) satisfies link FIFO and causality.

\subsection{Discretized link travel time model}

Traditionally, analytical DTA models can be classified into discrete time formulations and continuous time formulations. The discrete time models (see Merchant and Nemhauser, 1978; Huang and Lam, 2002; Lo and Szeto, 2002, for example) do not deal with continuous time variation, and discretize the 
time into a finite set of time intervals. The link travel time function in discrete DTA models are also formulated in discrete time space. On the other hand, those DTA models that are originally formulated in continuous time are either reformulated in discrete time later in order to solve them numerically or solved by numerical methods that involve discretising time (see Ran and Boyce, 1996; Lam and Huang, 1995; Nie and Zhang, 2010, for example). The reason for discretising time in the latter models is that there are no known methods for solving such complex continuous time network models analytically. Therefore, developing discretized link travel time models is a basic and important work for the solution of DTA models.

In discrete time DTA models, time $t$ is usually discretized into small time intervals, and we apply $\Delta t$ to denote the length of each interval. The average travel time of vehicles that enter the link during interval $(t, t+\Delta t]$ can be calculated by

$$
t_{a}(t)=\frac{\int_{U_{a}(t)}^{U_{a}(t+\Delta t)}\left[V_{a}^{-1}(v)-U_{a}^{-1}(v)\right] d v}{U_{a}(t+\Delta t)-U_{a}(t)},
$$

where $U_{a}^{-1}(\cdot)$ is the inverse function of $U_{a}(\cdot)$. The numerator of right hand side of Eq. (2) is equal to the shaded area in Fig. 1, and the denominator is the number of vehicles that enters link $a$ during this interval. If $\Delta t=0$, it follows immediately that $t_{a}(t)=\tau_{a}(t)$.

Definition 3 (Causality of discretized link travel time): Discretized link travel time satisfies causality if $t_{a}(t)$ depends on the traffic entering at time $t+\Delta t$ and earlier than $t+\Delta t$, but not on traffic entering later than $t+\Delta t$.

Proposition 1. If the road traffic flow satisfies causality, then the discretized link travel times calculated by Eq. (2) satisfies causality.

Proof. The discretized link travel time function defined by Eq.(2) can be equivalently reformulated as follows:

$$
t_{a}(t)=\frac{\int_{t}^{t+\Delta t} \tau_{a}(w) u_{a}(w) d w}{\int_{t}^{t+\Delta t} u_{a}(w) d w}
$$

If the road traffic flow satisfies causality, the time arguments of all variables (i.e., $\tau_{a}(w)$ and $u_{a}(w)$, $\forall w \leq t+\Delta t$ ) in Eq. (3) depend on the flows entering the link at time $t+\Delta t$ and earlier than $t+\Delta t$, but do not depend on the flows entering after time $t+\Delta t$. This completes the proof.

However, the discretized link travel time calculated by Eq. (2) may not satisfy link FIFO, even if the road traffic flow satisfies link FIFO. Below is an example that can illustrate this property.

Example 1. Violation of Link FIFO.

We assume the inflows last for two minutes, and the outflows occur during the period between 4 and 4.5 minutes. The time dependent cumulative inflows and outflows are given as follows:

$$
U_{a}(t)=60\left[(t-1)^{3}+1\right], 0 \leq t \leq 2,
$$


and

$$
V_{a}(t)= \begin{cases}0, & \text { if } t<4, \\ 240(t-4), & \text { if } 4 \leq t \leq 4.5 .\end{cases}
$$

Substituting Eqs. (4) and (5) into Eq. (1) and the numerator of the right hand side of Eq. (2), we have

$$
\tau_{a}(t)=\left[(t-1)^{3}+17\right] / 4-t,
$$

and

$$
\int\left[V_{a}^{-1}(v)-U_{a}^{-1}(v)\right] d v=\frac{1}{480} v^{2}+3 v-45 \sqrt[3]{(v / 60-1)^{4}} .
$$

Substituting Eq. (7) into Eq. (2), we can compute the link travel time $t_{a}(t)$ with an arbitrary interval length. If $t_{a}(t)$ satisfies link FIFO, $t+t_{a}(t)$ must be monotone with respect to time $t$. The profiles of $t+t_{a}(t)$ with various interval length are shown in Fig. 2. We can observe that the link travel time $t_{a}(t)$ will converge to $\tau_{a}(t)$ if $\Delta t \rightarrow 0$, and $t+\tau_{a}(t)$ is monotone when $\Delta t=0$. However, the results presented in Fig. 2 also show that $t+t_{a}(t)$ is not monotone when $\Delta t>0$ in this example. Therefore, the discretized link travel time calculated by Eq. (2) may not satisfy link FIFO, even if the road traffic flow satisfies link FIFO.

We state the following assumption and proposition to discuss the continuity property of the discretized link travel times.

Assumption 1. The cumulative inflows and outflows are continuous with respect to link inflows.

Proposition 2. Under Assumption 1, the link travel time function determined by Eq. (2) is continuous with respect to link inflows.

Proof. Since $U_{a}(t)=\int_{0}^{t} u_{a}(w) d w, U_{a}(t)$ and $U_{a}(t+\Delta t)$ are continuous with respect to link inflows, and hence the difference between two continuous functions is also continuous. Under Assumption 1, $V_{a}(t)$ is continuous with respect to link inflows. As the inverse of a continuous function is also continuous, the integrand is continuous. The definite integral in the numerator in Eq.(2) is also continuous. Therefore, the link travel time function determined by Eq. (2) is continuous.

In general, it is not possible to obtain inverse functions $U_{a}^{-1}(\cdot)$ and $V_{a}^{-1}(\cdot)$ as $U_{a}(\cdot)$ and $V_{a}(\cdot)$ are not strictly increasing in general. To overcome this problem, piecewise linear functions, such as the step function (SF) ( Lo and Szeto, 2002; Lian et al., 2007) and linear interpolation (LI) (Yperman, 2007) are in general applied to approximate the profiles of cumulative flows. In the following section, we will discuss three solution schemes to Eq. (2) based on SF and LI. These solution schemes can retain some desirable properties, such as FIFO, even when the discretisation is not refined and is "far" from the continuous limit. 


\section{Link travel time formulations based on cumulative flows}

\subsection{Notations}

The time period $T$ of interest is discretized into a finite set of time intervals, $K=\{k:=1,2, \cdots, \underline{K}\}$, where the length of each interval is $\delta$. The link of interest is assumed to be empty initially and positive inflows to this link occurs at a set of time intervals $K_{d}=\left\{k:=1,2, \cdots, \underline{K_{d}}\right\}$, where $\underline{K_{d}}<\underline{K}$. Note that we do not deal with the continuous time setting in this paper, since the DTA models are usually implemented on computers on the basis of time slices. The following notations will be adopted throughout this paper:

$U_{a}(k)$ the cumulative arrivals at link $a$ by the end of interval $k$.

$u_{a}(k)$ the inflow rate into link $a$ during interval $k$.

$V_{a}(k)$ the cumulative departures from link $a$ by the end of interval $k$.

$v_{a}(k)$ the outflow rate from link $a$ during interval $k$.

$y_{a}(k)$ the number of vehicles entering link $a$ during interval $k$.

$\mathbf{y}_{a}$ the vector of $\left(y_{a}(k), \forall k \in K_{d}\right)$.

$y_{a k}(l)$ the number of vehicles entering link $a$ during interval $k$ and exiting the link during interval $l$.

$Y_{a k}(l)$ the cumulative number of vehicles entering link $a$ during interval $k$ and exiting the link by the end of interval $l$.

$t_{a}(k)$ the average travel time for vehicles entering link $a$ during interval $k$.

$\mathbf{t}_{a}$ the vector of $\left(t_{a}(k), \forall k \in K_{d}\right)$.

$T_{a}(k)$ the total travel time of all the vehicles entering link $a$ during interval $k$.

$S_{a}(k)$ the outflow capacity of link $a$ during interval $k$.

With the assumption of a constant flow rate during each time interval, we have:

$$
\begin{gathered}
y_{a}(k)=\delta u_{a}(k)=U_{a}(k)-U_{a}(k-1), \text { and } \\
\delta v_{a}(k)=V_{a}(k)-V_{a}(k-1) .
\end{gathered}
$$

By definition, we also have:

$$
\begin{gathered}
y_{a}(k)=\sum_{l} y_{a k}(l), \text { and } \\
y_{a k}(l)=Y_{a k}(l)-Y_{a k}(l-1) .
\end{gathered}
$$

By the definition of $S_{a}(k)$, we have $S_{a}(k)=\int_{(k-1) \delta}^{k \delta} s_{a}(t) d t$, and $V_{a}(k)-V_{a}(k-1) \leq S_{a}(k)$. With the proposed treatment for DNL in Section 2.1, the cumulative inflow $U_{a}(k)$ will be strictly increasing, and 
$S_{a}(k)>0$ for all $k \in K$. According to Eq. (8), we have $y_{a}(k)>0$ for all $k \in K_{d}$ or $\mathbf{y}_{a}>\mathbf{0}$. In this paper, $\mathbf{y}_{a}>\mathbf{0}$ will be considered as a precondition in all link travel time models. In addition, $\tau_{a}^{0} / \delta$ denotes the number of intervals for vehicles traveling though link $a$ with free flow speed. Following the approximation in the CTM (see Daganzo, 1994, 1995a), we assume $\tau_{a}^{0} / \delta$ is an integer.

Definition 4 (Last departure interval). A last departure interval with respect to interval $k$ is defined as follows:

$$
m_{k}=\min \left\{l \mid U_{a}(k) \leq V_{a}(l), l \geq k+\tau_{a}^{0} / \delta\right\}
$$

Definition 5 (Critical outflow interval). A critical outflow interval with respect to interval $k$ is defined as follows:

$$
n_{k}=\min \left\{l \mid U_{a}(k) \leq V_{a}(l), l>k+\tau_{a}^{0} / \delta\right\}
$$

Remark 1. In Definition $4, m_{k}$ denotes the last interval by the end of which all the vehicles that enter link $a$ during interval $k$ have left the link. If $U_{a}(k)=V_{a}\left(k+\tau_{a}^{0} / \delta\right)$, then $m_{k}=k+\tau_{a}^{0} / \delta$ and $n_{k}=m_{k}+1$; Otherwise, $n_{k}=m_{k}$. The critical interval $n_{k}$ is used to determine the cumulative number of vehicles entering link $a$ during interval $k$ and exiting the link by each interval and to determine the last departure interval of all the vehicles entering link $a$ during interval $k$. By comparing $n_{k}$ and $m_{k}$, we can know whether the link is congested for vehicles entering link $a$ during interval $k$.

We state two propositions to describe some properties of $m_{k}$ and $n_{k}$, respectively. The two propositions will be used to analysis the properties of the link travel time functions concerned in this paper, and are given as follows:

Proposition 3. The last departure interval calculated by Eq. (12) implies that $U_{a}(k)>V_{a}\left(m_{k}-1\right)$ and $U_{a}(k)-V_{a}\left(m_{k}-1\right) \leq S_{a}\left(m_{k}\right)$.

Proof. Consider two cases: $m_{k}-1 \leq k-1+\tau_{a}^{0} / \delta$ and $m_{k}-1 \geq k+\tau_{a}^{0} / \delta$. If $m_{k}-1 \leq k-1+\tau_{a}^{0} / \delta$, we have: $U_{a}(k)=U_{a}(k-1)+y_{a}(k)>U_{a}(k-1) \geq V_{a}\left(k-1+\tau_{a}^{0} / \delta\right) \geq V_{a}\left(m_{k}-1\right)$; If $m_{k}-1 \geq k+\tau_{a}^{0} / \delta$, we assume $U_{a}(k) \leq V_{a}\left(m_{k}-1\right)$. As $U_{a}(k)$ can be equal to $V_{a}\left(m_{k}\right)$ by Eq. (12), we have $U_{a}(k)=V_{a}\left(m_{k}\right) \leq V_{a}\left(m_{k}-1\right)$ and $m_{k}-1 \geq m_{k}$. This is a contradiction, and therefore $U_{a}(k)>V_{a}\left(m_{k}-1\right)$ if $m_{k}-1 \geq k+\tau_{a}^{0} / \delta$. Combining two cases, we have $U_{a}(k)>V_{a}\left(m_{k}-1\right)$. Since the definition of $m_{k}$ implies that $U_{a}(k) \leq$ $V_{a}\left(m_{k}\right)$, we have $V_{a}\left(m_{k}-1\right)<U_{a}(k) \leq V_{a}\left(m_{k}\right)$, and $U_{a}(k)-V_{a}\left(m_{k}-1\right) \leq V_{a}\left(m_{k}\right)-V_{a}\left(m_{k}-1\right)$. The right hand side of the last inequality is not greater than the saturation flow $S_{a}\left(m_{k}\right)$ by definition. This completes the proof.

Proposition 4. The critical outflow interval calculated by Eq. (13) implies that $n_{k-1} \leq n_{k}$.

Proof. The definition of $n_{k}$ implies that $U_{a}(k) \leq V_{a}\left(n_{k}\right)$ and $n_{k}>k+\tau_{a}^{0} / \delta$, and Property 3 implies $U_{a}(k-1) \leq U_{a}(k) \leq V_{a}\left(n_{k}\right)$. Consequently, we have $U_{a}(k-1) \leq V_{a}\left(n_{k}\right)$, and $n_{k}>k-1+\tau_{a}^{0} / \delta$ Therefore, we have $n_{k} \geq \min \left\{l \mid U_{a}(k-1) \leq V_{a}(l), l>k-1+\tau_{a}^{0} / \delta\right\}$ where the right hand side equals $n_{k-1}$ by definition. This completes the proof. 


\subsection{The SF-type link travel time}

\subsubsection{Model formulation}

The actual link travel time of arrivals at time $t$ (i.e., $\left.u_{a}(t)\right)$ is the horizontal distance between the two cumulative curves as shown in Fig. 1. However, if time is discretized, taking a particular interval $k$ for example, there is no guarantee that the entire packet $y_{a}(k)$ will exit link $a$ during the same discretized time tick. Fig. 3 shows the profiles of the cumulative flows $U_{a}(k)$ and $V_{a}(k)$ of link a approximated by step functions. From this figure, we can observe that some portion of the packet has an estimated time of $\left(n_{k-1}-k\right) \delta$ whereas the other portions have longer estimated travel times. Their travel time differences and the estimated link travel time of the entire packet depend on the length of the discretized time interval. A smaller time interval length improves the accuracy of link travel time, but accompanying with a higher computational burden. As shown in Fig. 3, the traffic flow entering link $a$ during interval $k$ cannot exit the link until some time during interval $n_{k-1}$. Thus, the vehicles entering link $a$ during interval $k$ and exiting the link by interval $l$ is equal to zero if $l<n_{k-1}$. In addition, the entire packet $y_{a}(k)$ will completely exit the link before the end of interval $n_{k}$, and the cumulative flow $Y_{a k}(l)$ will have a constant value of $y_{a}(k)=U_{a}(k)-U_{a}(k-1)$ if $l \geq n_{k}$. For all $l \in\left[n_{k-1}, n_{k}\right)$, the cumulative flow $Y_{a k}(l)$ is equal to the difference between the cumulative outflow $V_{a}(l)$ and the cumulative inflow $U_{a}(k-1)$. In summary, the cumulative flow $Y_{a k}(l)$ can be formulated as follows:

$$
Y_{a k}(l)= \begin{cases}0, & \text { if } l<n_{k-1}, \\ V_{a}(l)-U_{a}(k-1), & \text { if } n_{k-1} \leq l<n_{k}, \\ U_{a}(k)-U_{a}(k-1), & \text { otherwise. }\end{cases}
$$

The number of vehicles entering link $a$ during interval $k$ and exiting the link during interval $l$ can be calculated by Eq. (11). The actual travel time for the sub-packet $y_{a k}(l)$ traveling on the link is $(l-k) \delta$ , and the total travel times of all vehicles in this sub-packet is $y_{a k}(l)(l-k) \delta$. Therefore, the total travel time $T_{a}(k)$ of the entire packet $y_{a}(k)$, which is the sum of the total travel time of each sub-packet, is equal to the shaded area in Fig. 3, and can be formulated as follows:

$$
T_{a}(k)=\sum_{l=1}^{K} y_{a k}(l)(l-k) \delta
$$

Substituting Eqs. (11) and (14) into Eq. (15), we have:

$$
T_{a}(k)=\left(n_{k}-k\right) \delta y_{a}(k)-\sum_{l=n_{k-1}}^{n_{k}-1} \delta\left[V_{a}(l)-U_{a}(k-1)\right] .
$$

The detailed derivation of $T_{a}(k)$ is given in Appendix A. Substituting Eq. (16) into Eq. (2), we have:

$$
t_{a}(k)=\frac{T_{a}(k)}{y_{a}(k)}=\left(n_{k}-k\right) \delta-\sum_{l=n_{k-1}}^{n_{k}-1} \delta\left[V_{a}(l)-U_{a}(k-1)\right] / y_{a}(k) .
$$




\subsubsection{Properties of the SF-type link travel time model}

Definition 6 (Link FIFO). The link FIFO condition is satisfied if and only if

$$
k^{\prime}>k^{\prime \prime} \Rightarrow k^{\prime} \delta+t_{a}\left(k^{\prime}\right) \geq k^{\prime \prime} \delta+t_{a}\left(k^{\prime \prime}\right), \forall k^{\prime}, k^{\prime \prime} \in K_{d} .
$$

In other words, the link FIFO condition is satisfied if vehicles that enter the link earlier will leave sooner.

Proposition 5. The dynamic link travel time calculated by Eq. (17) satisfies link FIFO.

Proof. By considering discrete time intervals $k$ and $k+1$, we have this link FIFO condition: Link FIFO is satisfied if and only if

$$
k+1>k \Rightarrow(k+1) \delta+t_{a}(k+1) \geq k \delta+t_{a}(k) .
$$

The first inequality can be omitted because it is always true, and the second inequality can be re-expressed as

$$
t_{a}(k+1)-t_{a}(k)+\delta \geq 0 .
$$

The second inequality must hold because:

$$
\begin{aligned}
\frac{t_{a}(k+1)-t_{a}(k)+\delta}{\delta} & =\left\{\left(n_{k+1}-k-1\right)-\sum_{l=n_{k}}^{n_{k+1}-1}\left[V_{a}(l)-U_{a}(k)\right] / y_{a}(k+1)\right\} \\
& -\left\{\left(n_{k}-k\right)-\sum_{l=n_{k-1}}^{n_{k}-1}\left[V_{a}(l)-U_{a}(k-1)\right] / y_{a}(k)\right\}+1 \\
& \left.=n_{k+1}-n_{k}+\sum_{l=n_{k-1}}^{n_{k}-1}\left[V_{a}(l)-U_{a}(k-1)\right] / y_{a}(k)\right\}-\sum_{l=n_{k}}^{n_{k+1}-1}\left[V_{a}(l)-U_{a}(k)\right] / y_{a}(k+1) \\
& \geq n_{k+1}-n_{k}-\sum_{l=n_{k}}^{n_{k+1}-1}\left[V_{a}(l)-U_{a}(k)\right] / y_{a}(k+1) \\
& =\sum_{l=n_{k}}^{n_{k+1}-1}\left\{y_{a}(k+1)-\left[V_{a}(l)-U_{a}(k)\right]\right\} / y_{a}(k+1) \\
& \left.=\sum_{l=n_{k}}^{n_{k+1}-1}\left[U_{a}(k+1)-V_{a}(l)\right]\right\} / y_{a}(k+1) \\
& \geq 0 .
\end{aligned}
$$

This completes the proof.

Proposition 6. The dynamic link travel time calculated by Eq. (17) satisfies causality.

Proof. Eq. (8) and the definition of $n_{k}$ in Eq. (13) imply that both $U_{a}(k)$ and $n_{k}$ depend on the variables $y_{a}\left(k^{\prime}\right), k^{\prime}=1,2, . ., k$, respectively. Moreover, the variable $V_{a}(l)$ (for all $l<n_{k}$ ) satisfies $V_{a}(l) \leq U_{a}(k)$, and then $V_{a}(l)$ also depends on the variables $y_{a}\left(k^{\prime}\right), k^{\prime}=1,2, . ., k$. In summary, the time argument of all variables in Eq. (17) depend on the flows entering the link before the end of interval $k$, but do not depend on the flows entering after the end of interval $k$. 
Propositions 5 and 6 show that the SF-type link travel model satisfies link FIFO and causality. This implies that the solutions of DTA models using the SF-type travel time approximation (see Lo and Szeto, 2002; Lian et al., 2007, for example) satisfy the link FIFO and causality properties.

It is also important to investigate continuity and monotonicity of the SF-type link travel time function, because the existence and uniqueness of the solutions of DTA models depend on these properties.

Proposition 7. Under Assumption 1, the traffic flow $y_{a k}(l)$ is continuous with respect to link inflow $\mathbf{y}_{a}$.

Proof. In Eq. (14), we can consider two cases: (i) $U_{a}(k-1)<V_{a}\left(n_{k-1}\right)$ and $U_{a}(k)<V_{a}\left(n_{k}\right)$. The definition of $n_{k}$ implies that a very small change of inflows will not change $n_{k-1}$ and $n_{k}$. In this case, $Y_{a k}(l)$ is continuous with respect to $U_{a}(k)$ and $V_{a}(k)$. (ii) Boundary conditions. If $U_{a}(k-1)=V_{a}\left(n_{k-1}\right)$, we have $Y_{a k}\left(n_{k-1}\right)=Y_{a k}\left(n_{k-1}-1\right)=0$. Similarly, if $U_{a}(k)=V_{a}\left(n_{k}\right)$, we have $Y_{a k}\left(n_{k}\right)=U_{a}(k)-$ $U_{a}(k-1)=V_{a}\left(n_{k}\right)-U_{a}(k-1)$. Those imply two adjacent pieces in Eq. (14) give the same value at the boundary. Therefore, the two cases imply that $Y_{a k}(l)$ is continuous with respect to $U_{a}(k)$ and $V_{a}(k)$. Under Assumption 1, $U_{a}(k)$ and $V_{a}(k)$ are continuous with respect to $\mathbf{y}_{a}$. Hence, $Y_{a k}(l)$ is continuous with respect to $\mathbf{y}_{a}$. Since Eq. (11) implies $y_{a k}(l)$ is continuous with respect to $Y_{a k}(l), y_{a k}(l)$ is also continuous with respect to $\mathbf{y}_{a}$. This completes the proof.

Proposition 8. Under Assumption 1, the actual link travel time $\mathbf{t}_{a}$ calculated by Eq. (17) is a continuous function of $\mathbf{y}_{a}$.

Proof. Substituting Eq. (15) into Eq. (17), we have $t_{a}(k)=\sum_{l=1}^{K} y_{a k}(l)(l-k) \delta / \sum_{l=1}^{K} y_{a k}(l)$. According to Proposition $7, y_{a k}(l)$ is continuous with respect to $\mathbf{y}_{a}$, and $\mathbf{t}_{a}$ is also continuous with respect to $\mathbf{y}_{a}$.

Lemma 1 ( Chen, 1999, Theorem 2.9). Suppose that $\mathbf{c}(\mathbf{u})$ is continuously differentiable on $\Omega$ and the Jacobian matrix $\nabla \mathbf{c}(\mathbf{u})$ is positive semidefinite (or positive definite). Then $\mathbf{c}(\mathbf{u})$ is monotone (or strictly monotone).

Assumption 2. The link travel time function is separable (i.e., the travel time on a link is unaffected by the flows on all the other links in the network).

Proposition 9. Under Assumption 2, if $U_{a}(k)-V_{a}\left(m_{k}-1\right)<S_{a}\left(m_{k}\right), \forall k \in K_{d}$, then the dynamic link travel time $\mathbf{t}_{a}\left(\mathbf{y}_{a}\right)$ calculated by Eq. (17) is a differentiable function of $\mathbf{y}_{a}$.

The proof is presented in Appendix B.

Proposition 10. Under Assumption 2, the dynamic link travel time $\mathbf{t}_{a}\left(\mathbf{y}_{a}\right)$ calculated by Eq. (17) is a monotone function of $\mathbf{y}_{a}$.

The proof is presented in Appendix C.

As shown in Examples 2 and 3 in the numerical study section, $\mathbf{t}_{a}\left(\mathbf{y}_{a}\right)$ may not be strictly monotonic. 


\subsection{LI-type link travel time}

\subsubsection{Model formulation}

Following Yperman (2007), linear interpolation can be applied to calculate the cumulative flows during a particular time interval as shown in Fig. 4.

The cumulative flows at time $(k+\mu) \delta$ can be calculated as follows:

$$
\left\{\begin{array}{c}
U_{a}(k+\mu)=(1-\mu) U_{a}(k)+\mu U_{a}(k+1), \\
V_{a}(k+\mu)=(1-\mu) V_{a}(k)+\mu V_{a}(k+1) .
\end{array}\right.
$$

where $\mu \in[0,1]$.

If the cumulative outflow at time $(k+\mu) \delta$ is predetermined, then we can calculate $\mu$ as follows:

$$
\mu=\frac{V_{a}(k+\mu)-V_{a}(k)}{V_{a}(k+1)-V_{a}(k)} .
$$

As shown in Fig. 5, the flow packet $y_{a k}\left(n_{k}\right)$ exits link $a$ accompanying with some flows entering the link after interval $k$. In particular, at time $\left(n_{k}-1+\mu_{k}\right) \delta$, where $\mu_{k}$ is a cumulative outflow parameter associated with interval $k$, the flows entering link $a$ before interval $k+1$ have completely exited the link, and inflow belonging to interval $k+1$ starts leaving away the link. The exact time for this occurrence can be expressed as follows:

$$
\mu_{k}=\frac{U_{a}(k)-V_{a}\left(n_{k}-1\right)}{V_{a}\left(n_{k}\right)-V_{a}\left(n_{k}-1\right)} .
$$

This can be deduced by Eq. (20) and $U_{a}(k)=V_{a}\left(n_{k}-1+\mu_{k}\right)$.

Theorem 1. $\mu_{k}$ has the following properties

1. $0 \leq \mu_{k} \leq 1$.

2. $\mu_{k-1}<\mu_{k}$ if $n_{k-1}=n_{k}$.

3. $\mu_{k}>0$ and $\mu_{k-1}<1$ if $n_{k-1}=n_{k}$.

Proof. The definition of $n_{k}$ implies $n_{k} \geq k+\tau_{a}^{0} / \delta+1$ and $U_{a}(k) \leq V_{a}\left(n_{k}\right)$. If $n_{k}>k+\tau_{a}^{0} / \delta+1$, the definition of $n_{k}$ implies $V_{a}\left(n_{k}-1\right)<U_{a}(k)$. If $n_{k}=k+\tau_{a}^{0} / \delta+1$, Property 4 implies that $U_{a}(k) \geq$ $V_{a}\left(k+\tau_{a}^{0} / \delta\right)$, and we have: $V_{a}\left(n_{k}-1\right)=V_{a}\left(k+\tau_{a}^{0} / \delta\right) \leq U_{a}(k)$. If $V_{a}\left(n_{k}-1\right)=U_{a}(k), \mathbf{y}_{a}>\mathbf{0}$ implies $U_{a}(k)<V_{a}\left(n_{k}\right)$; otherwise, $V_{a}\left(n_{k}-1\right)<U_{a}(k)$. In summary, we have: $V_{a}\left(n_{k}-1\right) \leq U_{a}(k) \leq V_{a}\left(n_{k}\right)$, $V_{a}\left(n_{k}-1\right)<V_{a}\left(n_{k}\right)$, and $0 \leq \mu_{k}=\frac{U_{a}(k)-V_{a}\left(n_{k}-1\right)}{V_{a}\left(n_{k}\right)-V_{a}\left(n_{k}-1\right)} \leq 1$.

If $n_{k-1}=n_{k}$, we have: 


$$
\begin{aligned}
\mu_{k}-\mu_{k-1} & =\frac{U_{a}(k)-V_{a}\left(n_{k}-1\right)}{V_{a}\left(n_{k}\right)-V_{a}\left(n_{k}-1\right)}-\frac{U_{a}(k-1)-V_{a}\left(n_{k-1}-1\right)}{V_{a}\left(n_{k-1}\right)-V_{a}\left(n_{k-1}-1\right)} \\
& =\frac{U_{a}(k)-V_{a}\left(n_{k}-1\right)}{V_{a}\left(n_{k}\right)-V_{a}\left(n_{k}-1\right)}-\frac{U_{a}(k-1)-V_{a}\left(n_{k}-1\right)}{V_{a}\left(n_{k}\right)-V_{a}\left(n_{k}-1\right)} \\
& =\frac{U_{a}(k)-U_{a}(k-1)}{V_{a}\left(n_{k}\right)-V_{a}\left(n_{k}-1\right)} \\
& =\frac{y_{a}(k)}{V_{a}\left(n_{k}\right)-V_{a}\left(n_{k}-1\right)} \\
& >0 .
\end{aligned}
$$

Therefore, $\mu_{k-1}<\mu_{k}$ if $n_{k-1}=n_{k}$. Moreover, by property 1 in this proposition, $\mu_{k-1} \geq 0$ and $\mu_{k} \leq 1$. These two conditions combine with $\mu_{k}>\mu_{k-1}$ giving $\mu_{k}>\mu_{k-1} \geq 0$, and $\mu_{k-1}<\mu_{k} \leq 1$, which imply $\mu_{k}>0$ and $\mu_{k-1}<1$ respectively. This completes the proof.

Figure 5 also shows that all the vehicles or flows $y_{a}(k)$ entering link $a$ during interval $k$ can leave the link in different time intervals, and hence all the vehicles have different link travel times. Their total travel time is represented by the shaded area in Fig. 5. This shaded area can be decomposed to two areas: the area representing the total travel time of these vehicles traveling during interval $k, \frac{1}{2} \delta y_{a}(k)$, and the area representing the total travel time of these vehicles traveling after interval $k$. The latter can further be decomposed into many trapezoids. To express the area of each trapezoid mathematically, we define the following notations:

$$
\alpha_{l}= \begin{cases}\mu_{k-1}, & \text { if } l \leq n_{k-1} \\ 0, & \text { otherwise }\end{cases}
$$

and

$$
\beta_{l}= \begin{cases}1, & \text { if } l<n_{k} \\ \mu_{k}, & \text { otherwise }\end{cases}
$$

Then, the area of the trapezoid for subflow packet $l$ is formulated as $\frac{1}{2} y_{a k}(l)\left[\left(l-k+\alpha_{l}-1\right) \delta+(l-k+\right.$ $\left.\left.\beta_{l}-1\right) \delta\right]$, where $\left(l-k+\alpha_{l}-1\right) \delta$ and $\left(l-k+\beta_{l}-1\right) \delta$ are the length of the bottom and top bases respectively. The total travel time of all the vehicles entering link $a$ during interval $k$ can be mathematically expressed as follows:

$$
\hat{T}_{a}(k)=\frac{1}{2} \delta y_{a}(k)+\frac{1}{2} \sum_{l=1}^{\underline{K}} y_{a k}(l)\left[\left(l-k+\alpha_{l}-1\right) \delta+\left(l-k+\beta_{l}-1\right) \delta\right] .
$$

Equation (24) can be simplified as:

$$
\begin{aligned}
\hat{T}_{a}(k) & =\sum_{l=1}^{\underline{K}} y_{a k}(l)(l-k) \delta+\frac{1}{2} \sum_{l=1}^{\underline{K}} y_{a k}(l)\left(\alpha_{l}+\beta_{l}-2\right) \delta+\frac{1}{2} \delta y_{a}(k) \\
& =T_{a}(k)+\frac{1}{2} \sum_{l=1}^{\underline{K}} y_{a k}(l)\left(\alpha_{l}+\beta_{l}-1\right) \delta .
\end{aligned}
$$


Let $\triangle T_{a}(k)$ be the difference of the LI-type total travel time from the SF-type total travel time. Then, we have:

$$
\triangle T_{a}(k)=\hat{T}_{a}(k)-T_{a}(k)=\frac{1}{2} \sum_{l=1}^{\underline{K}} y_{a k}(l)\left(\alpha_{l}+\beta_{l}-1\right) \delta .
$$

Substituting Eqs. (11), (22), and (23) into Eq. (26), we have:

$$
\Delta T_{a}(k)= \begin{cases}\frac{1}{2} \delta\left(\mu_{k-1}+\mu_{k}-1\right) y_{a}(k), & \text { if } n_{k-1}=n_{k}, \\ \frac{1}{2} \delta\left[\mu_{k-1} y_{a k}\left(n_{k-1}\right)+\left(\mu_{k}-1\right) y_{a k}\left(n_{k}\right)\right], & \text { otherwise. }\end{cases}
$$

Since $y_{a k}\left(n_{k-1}\right)=y_{a k}\left(n_{k}\right)=y_{a}(k)$ if $n_{k-1}=n_{k}$, Eq. (27) can be rewritten as:

$$
\triangle T_{a}(k)=\frac{1}{2} \delta\left[\mu_{k-1} y_{a k}\left(n_{k-1}\right)+\left(\mu_{k}-1\right) y_{a k}\left(n_{k}\right)\right] .
$$

Therefore, the LI-type link travel time $\hat{t}_{a}(k)$ can be stated as follows:

$$
\hat{t}_{a}(k)=\frac{\hat{T}_{a}(k)}{y_{a}(k)}=t_{a}(k)+\frac{1}{2} \delta\left[\mu_{k-1} y_{a k}\left(n_{k-1}\right)+\left(\mu_{k}-1\right) y_{a k}\left(n_{k}\right)\right] / y_{a}(k)
$$

where $t_{a}(k)$ is the SF-type link travel time defined by Eq. (17).

\subsubsection{Properties of LI-type link travel time}

Definition 7 (Link strong FIFO (SFIFO)). The link SFIFO condition is satisfied if and only if

$$
k^{\prime}>k^{\prime \prime} \Rightarrow k^{\prime} \delta+t_{a}\left(k^{\prime}\right)>k^{\prime \prime} \delta+t_{a}\left(k^{\prime \prime}\right), \forall k^{\prime}, k^{\prime \prime} \in K_{d} .
$$

Proposition 11. The dynamic link travel time calculated by Eq. (29) satisfies the link SFIFO condition.

The proof is presented in Appendix D.

Proposition 12. Under Assumption 1, the link travel time $\hat{\mathbf{t}}_{a}$ calculated by Eq. (29) is a continuous function of $\mathbf{y}_{a}$.

Proof. Equation (14) implies $Y_{a k}\left(n_{k}-1\right)=V_{a}\left(n_{k}-1\right)-U_{a}(k-1)$ and $Y_{a k}\left(n_{k}\right)=U_{a}(k)-U_{a}(k-1)$. Using Eq. (11), we have: $y_{a k}\left(n_{k}\right)=Y_{a k}\left(n_{k}\right)-Y_{a k}\left(n_{k}-1\right)=U_{a}(k)-V_{a}\left(n_{k}-1\right)$. Therefore, Eq. (21) can be re-written as: $\mu_{k}=\frac{U_{a}(k)-V_{a}\left(n_{k}-1\right)}{\left(V_{a}\left(n_{k}\right)-U_{a}(k)\right)+\left(U_{a}(k)-V_{a}\left(n_{k}-1\right)\right)}=y_{a k}\left(n_{k}\right) /\left[y_{a k+1}\left(n_{k}\right)+y_{a k}\left(n_{k}\right)\right]$. According to Proposition $7, \frac{1}{2} \delta\left[\mu_{k-1} y_{a k}\left(n_{k-1}\right)+\left(\mu_{k}-1\right) y_{a k}\left(n_{k}\right)\right] / y_{a}(k)$ is continuous with respect to $\mathbf{y}_{a}$. Proposition 8 shows that $\mathbf{t}_{a}$ is a continuous function of $\mathbf{y}_{a}$. Therefore, $\hat{\mathbf{t}}_{a}$ is a continuous function of $\mathbf{y}_{a}$.

Proposition 13. The dynamic link travel time calculated by Eq. (29) does not satisfy causality.

Proof. Before the calculation of the LI-type link travel time, the parameter $\mu_{k}$ should be predetermined. We can observe from Eq. (21) that the value of $V_{a}\left(n_{k}\right)$ directly determines the value of $\mu_{k}$. Since $V_{a}\left(n_{k}\right) \geq U_{a}(k)$, the cumulative outflow by interval $n_{k}$ consists of the flows entering link $a$ during interval $k$ or earlier than interval $k$, and also may consist of the flows entering link $a$ after the end of interval $k$. This implies that the value of $\mu_{k}$ not only depends on the flows entering link $a$ during interval $k$ or earlier than interval $k$, but also may depend on the flows entering link $a$ after the end of interval $k$. Therefore, the LI-type link travel time does not satisfy causality. 


\subsection{Modified LI-type link travel time}

Since the LI-type link travel time does not satisfy causality due to the calculation of $\mu_{k}$ (see Proposition 13), we propose a new method to calculate the cumulative outflow parameter denoted by

$$
\bar{\mu}_{k}=\frac{U_{a}(k)-V_{a}\left(n_{k}-1\right)}{S_{a}\left(n_{k}\right)},
$$

where $S_{a}\left(n_{k}\right)$ is the outflow capacity of link $a$ at interval $n_{k}$. Note that the outflow capacity of link $a$ at each interval is predetermined before network loading, and hence independent of link inflows. By definition, we have $S_{a}\left(n_{k}\right) \geq V_{a}\left(n_{k}\right)-V_{a}\left(n_{k}-1\right)$, and therefore $\bar{\mu}_{k} \leq \mu_{k}$. Since $U_{a}(k)-V_{a}\left(n_{k}-1\right)=$ $y_{a k}\left(n_{k}\right)$ (see the proof of Proposition 12 for the details), we have $\bar{\mu}_{k}=y_{a k}\left(n_{k}\right) / S_{a}\left(n_{k}\right)$.

Theorem 2. $\bar{\mu}_{k}$ has the following properties:

1. $0 \leq \bar{\mu}_{k} \leq 1$.

2. $\bar{\mu}_{k-1}<\bar{\mu}_{k}$ if $n_{k-1}=n_{k}$.

3. $\bar{\mu}_{k}>0$ and $\bar{\mu}_{k-1}<1$ if $n_{k-1}=n_{k}$.

Proof. Since $V_{a}\left(n_{k}\right)-V_{a}\left(n_{k}-1\right) \leq S_{a}\left(n_{k}\right)$, and the proof of Theorem 1 shows that $V_{a}\left(n_{k}-1\right) \leq U_{a}(k) \leq$ $V_{a}\left(n_{k}\right)$, we can obtain $0 \leq U_{a}(k)-V_{a}\left(n_{k}-1\right) \leq V_{a}\left(n_{k}\right)-V_{a}\left(n_{k}-1\right) \leq S_{a}\left(n_{k}\right)$. Therefore, $0 \leq \bar{\mu}_{k} \leq 1$ is satisfied. The proofs of the properties 2 and 3 in this proposition are similar to those in Theorem 1.

With the redefined cumulative outflow parameter in Eq. (30), it is easy for us to obtain a modified LI-type (MLI-type) link travel time function

$$
\bar{t}_{a}(k)=t_{a}(k)+\frac{1}{2} \delta\left[\bar{\mu}_{k-1} y_{a k}\left(n_{k-1}\right)+\left(\bar{\mu}_{k}-1\right) y_{a k}\left(n_{k}\right)\right] / y_{a}(k) .
$$

Proposition 14. The dynamic link travel time calculated by Eq. (31) satisfies the link SFIFO condition.

Proof. The redefined cumulative outflow parameter $\bar{\mu}_{k}$ has the same properties with $\mu_{k}$, and therefore the proof of Proposition 11 implies that the MLI-type link travel time satisfies the link SFIFO condition.

Proposition 15. The dynamic link travel time calculated by Eq. (31) satisfies causality.

Proof. The predetermined parameter $\bar{\mu}_{k}$ in Eq. (30) depends on the flow-dependent variables $U_{a}(k)$ and $V_{a}\left(n_{k}-1\right)$ and the flow independent variable $S_{a}\left(n_{k}\right)$. Proposition 5 shows that $n_{k}$ and $t_{a}(k)$ only depend on the parameter $y_{a}\left(k^{\prime}\right)$ from 1 to $k$. Hence, $\bar{\mu}_{k}, y_{a k}\left(n_{k-1}\right)$ and $y_{a k}\left(n_{k}\right)$ does not depend on the flows entering link $a$ after the end of interval $k$. Therefore, the time arguments of all variables in Eq. (31) only depend on the flows entering the link before the end of interval $k$, but do not depend on the flows entering after the end of interval $k$. This completes the proof. 
Propositions 14 and 15 show that the MLI-type travel time model satisfies Link SFIFO and causality. It is also interesting and important to investigate continuity and monotonicity of this proposed link travel time function.

Proposition 16. Under Assumption 1, the link travel time $\overline{\mathbf{t}}_{a}$ calculated by Eq. (31) is a continuous function of $\mathbf{y}_{a}$.

Proof. Equation (30) can be reformulated as: $\bar{\mu}_{k}=y_{a k}\left(n_{k}\right) / S_{a}\left(n_{k}\right)$, where $S_{a}\left(n_{k}\right)$ is predetermined and independent with $\mathbf{y}_{a}$. According to Proposition $7, \frac{1}{2} \delta\left[\bar{\mu}_{k-1} y_{a k}\left(n_{k-1}\right)+\left(\bar{\mu}_{k}-1\right) y_{a k}\left(n_{k}\right)\right] / y_{a}(k)$ is continuous with respect to $\mathbf{y}_{a}$. Proposition 8 shows that $\mathbf{t}_{a}$ is a continuous function of $\mathbf{y}_{a}$. Therefore, $\overline{\mathbf{t}}_{a}$ is a continuous function of $\mathbf{y}_{a}$.

Proposition 17. Under Assumption 2, if $U_{a}(k)-V_{a}\left(m_{k}-1\right)<S_{a}\left(m_{k}\right), \forall k \in K_{d}$, the dynamic link travel time $\overline{\mathbf{t}}_{a}\left(\mathbf{y}_{a}\right)$ calculated by Eq. (31) is a differentiable function of $\mathbf{y}_{a}$.

The proof is presented in Appendix E.

Proposition 18. Under Assumption 2, the link travel time $\overline{\mathbf{t}}_{a}\left(\mathbf{y}_{a}\right)$ calculated by Eq. (31) is a monotone function of $\mathbf{y}_{a}$.

The proof is similar to that of Proposition 10.

Definition 8 (Link Congestion). The link congestion condition can be mathematically expressed as follows:

$$
U_{a}(k)>V_{a}\left(k+\tau_{a}^{0} / \delta\right), \forall k \in K_{d}
$$

The link congestion condition implies that the travel time for all vehicles will be greater than the free flow travel time on this link. Since the time period of interest is discretized into a finite set of time intervals, this condition requires the inflows belonging to interval $k$ cannot completely leave the link before the time $k \delta+\tau_{a}^{0}$. Therefore, this condition also implies that $m_{l}=n_{l}$ for all $l \in K_{d}$.

Proposition 19. If link $a$ is under the congestion condition, then $\bar{\mu}>0$, where $\bar{\mu}=\left(\bar{\mu}_{k}, k \in K_{d}\right)$ is the vector of cumulative outflow parameters.

Proof. For a particular interval $k$, assume $\bar{\mu}_{k}=0$. The definition of $\bar{\mu}_{k}$ implies $U_{a}(k)=V_{a}\left(n_{k}-1\right)$. Since link $a$ is under the congestion condition, we have $V_{a}\left(n_{k}-1\right)=U_{a}(k)>V_{a}\left(k+\tau_{a}^{0} / \delta\right)$. Therefore, $n_{k}-1>k+\tau_{a}^{0} / \delta$ and $V_{a}\left(n_{k}-1\right) \geq U_{a}(k)$ are consequently satisfied, and hence $n_{k}-1 \geq \min \left\{l \mid U_{a}(k) \leq\right.$ $\left.V_{a}(l), l>k+\tau_{a}^{0} / \delta\right\}=n_{k}$. This is a contradiction. Therefore, $\bar{\mu}_{k}>0$ should be satisfied. This completes the proof.

Proposition 20. Under Assumption 2, if link $a$ is under the congestion condition, the link travel time $\overline{\mathbf{t}}_{a}\left(\mathbf{y}_{a}\right)$ calculated by Eq. (31) is a strictly monotone function of $\mathbf{y}_{a}$.

The proof is presented in Appendix F. 


\subsection{Comparison of various types of travel times}

This section aims at comparing the SF-type, the LI-type, and the MLI-type travel times and their properties. We first estimate their travel time differences. From Eq. (29), the difference or the gap between the SF-type and LI-type travel times $\Delta \hat{t}_{a}(k)$ can be obtained as follows:

$$
\begin{aligned}
\Delta \hat{t}_{a}(k) & =\hat{t}_{a}(k)-t_{a}(k) \\
& =\frac{1}{2} \delta\left[\mu_{k-1} y_{a k}\left(n_{k-1}\right)+\left(\mu_{k}-1\right) y_{a k}\left(n_{k}\right)\right] / y_{a}(k) \\
& =\frac{1}{2} \delta \mu_{k-1} y_{a k}\left(n_{k-1}\right) / y_{a}(k)-\frac{1}{2} \delta\left(1-\mu_{k}\right) y_{a k}\left(n_{k}\right) / y_{a}(k) .
\end{aligned}
$$

Since $\mu_{k} \in[0,1]$ and $y_{a k}(l) / y_{a}(k) \in[0,1], \forall k \in K_{d}, l \in K$, we have:

$$
\Delta \hat{t}_{a}(k) \leq \frac{1}{2} \delta \mu_{k-1} y_{a k}\left(n_{k-1}\right) / y_{a}(k) \leq \frac{1}{2} \delta
$$

and

$$
\Delta \hat{t}_{a}(k) \geq-\frac{1}{2} \delta\left(1-\mu_{k}\right) y_{a k}\left(n_{k}\right) / y_{a}(k) \geq-\frac{1}{2} \delta .
$$

Eqs. (33) and (34) define the range of the gap between the SF-type and LI-type link travel times which is $\left[-\frac{1}{2} \delta, \frac{1}{2} \delta\right]$. Similar with the above derivation, we can also obtain the range of the gap between the SF-type and MLI-type link travel times denoted by $\Delta \bar{t}_{a}(k)$, which is $\left[-\frac{1}{2} \delta, \frac{1}{2} \delta\right]$. This result indicates that the difference of the LI-type (MLI-type) link travel time from the SF-type link travel time is within

half interval length $\frac{1}{2} \delta$. If $\delta \rightarrow 0$, the three types of link travel time functions will converge to the same function. This implies that the subtle difference between the three algorithms in the paper will diminish as the discrete time interval converges to zero. However, it is computationally too costly to work with discrete time intervals approaching zero in practice for large scale DTA network modeling. Larger discrete time intervals will be needed in practice and in that case the differences between the methods in the paper are significant and important.

Next, we compare the properties of the three types of link travel times and their properties are summarized in Table 1 . In the table, we can see that all the three types of travel times satisfy link FIFO and continuity. Comparing the three types of travel times, only the LI-type travel time does not satisfy causality. Disobeying causality implies that the speed and travel time of a vehicle on a link may be affected by the speed of vehicles behind. This is unrealistic for the observed traffic. The SF-type model has a simpler calculation, and can be extended to calculate route travel time by using cumulative route flows, whereas, the MLI-type model has better properties than the SF-type model such as satisfying Link SFIFO and strictly monotone under some assumptions.

\subsection{Model accuracy estimation}

To estimate the theoretical value of link travel time, we set a very short interval length (e.g., 0.1 second in this paper), and run the DNL model to obtain the SF-type link travel time (denoted by $\check{t}_{a}(\iota)$ ) and inflow 
(denoted by $\check{y}_{a}(\iota)$ ), where $\iota$ is the index of a particular short interval. For a given interval length (e.g., $\delta)$, the theoretical value of link travel time during a particular interval $k$ can be numerically calculated as follows:

$$
t t_{a}(k)=\frac{\sum_{\iota \in K^{\delta}(k)} \check{y}_{a}(\iota) \check{t}_{a}(\iota)}{y_{a}(k)},
$$

where $t t_{a}(k)$ is the estimated theoretical link travel time at interval $k, K^{\delta}(k)$ is the set of short intervals that belong to the time period of $((k-1) \delta, k \delta]$.

The estimated theoretical link travel time is used to evaluate the accuracy of the presented link travel time models and the accuracy is measured by calculation errors. The calculation error of a model for a particular interval is defined as the difference between the estimated travel time by a model and the theoretical link travel time at that interval. Using the SF-type link travel time as an example, the calculation error of the link travel time at a given interval $k \in K_{d}$ can be mathematically represented as follows:

$$
e_{a}(k)=t_{a}(k)-t t_{a}(k)
$$

For measuring the average error of the whole study period for a particular model, there are two categories: mean absolute error (MAE) and mean percentage error (MPE). The MAE and MPE can be mathematically expressed as follows:

$$
\begin{gathered}
M A E=\frac{\sum_{k \in K_{d}}\left|e_{a}(k)\right|}{\underline{K_{d}}}=\frac{\sum_{k \in K_{d}}\left|t_{a}(k)-t t_{a}(k)\right|}{\underline{K_{d}}}, \text { and } \\
M P E=\frac{\sum_{k \in K_{d}}\left|e_{a}(k)\right| / t t_{a}(k)}{\underline{K_{d}}} \times 100=\frac{\sum_{k \in K_{d}}\left|t_{a}(k)-t t_{a}(k)\right| / t t_{a}(k)}{\underline{K_{d}}} \times 100 .
\end{gathered}
$$

$\sum_{k \in K_{d}}\left|t_{a}(k)-t t_{a}(k)\right|$ is the total absolute calculation error (in seconds) and $\underline{K_{d}}$ is the number of periods considered. Therefore, Eq. (37) gives MAE (in seconds). In Eq. (38), MPE is defined as the average of the relative absolute calculation error of each discretized interval and expressed in terms of per 100 .

The calculation error of link travel time is highly related to interval length. We also propose a new method to evaluate the average calculation error, defined as interval relative error (IRE). IRE can be mathematically expressed as follows:

$$
I R E=\frac{M A E}{\delta} \times 100=\frac{\sum_{k \in K_{d}}\left|t_{a}(k)-t t_{a}(k)\right|}{\underline{K_{d}} \delta} \times 100 .
$$

In Eq. (39), IRE is defined as the ratio of MAE (in seconds) to the length of a discretized interval (in seconds) and expressed in terms of per 100. This IRE can be viewed as the total absolute calculation error divided by the total length of the study period and is expressed in percent. 


\section{$4 \quad$ Numerical Examples}

Several DNL models, in which traffic is propagated through the network along the assigned routes, can be applied to generate both link and route cumulative flows. The presented link travel time models use the cumulative link flows to calculate time-dependent link travel times. In the past decade, the CTM originally proposed by Daganzo $(1994,1995$ a) was popularly applied to calculate travel times for DTA models (e.g., Ziliaskopoulos, 2000; Lo and Szeto, 2002; Szeto and Lo, 2004; Lian et al., 2007). Only recently, a combination of Daganzo's CTM with a triangular fundamental diagram and cumulative curves (Newell, 1993) was constructed by Yperman (2007), resulting in the link transmission model (LTM). Because each whole link can be treated as one cell, the LTM's computational efficiency is much higher than that of classic numerical solution schemes for the LWR model, whilst retaining the same accuracy. Yperman (2007) employed a simple example to demonstrate that the LTM has a higher accuracy in modeling traffic dynamics than CTM. To illustrate the properties of the presented travel time models, the DNL model employed in this paper was the LTM (see Yperman, 2007, for the details). We only consider that vehicles travel on a single link with a bottleneck at the link's exit. The bottleneck will cause congestion.

Example 2. With given inflows and outflows.

In this example, interval length $\delta$ is 10 seconds; inflow capacity is 12 vehicles per interval; the outflow capacity is 7 vehicles per interval, and the free flow travel time is 3 intervals (i.e., 30 seconds). The study period consists of 10 intervals, and only the first 6 intervals have inflows. The inflows, cumulative inflows, outflows, and cumulative outflows in each interval are given in Table 2. The profiles of the SF-type and LI-type cumulative flows are displayed in Fig. 6. The cumulative flows by each interval are applied to calculate link travel times by the proposed approaches with the results listed in Table 3 .

The link travel times given in Table 3 demonstrate that the three types of link travel time models may obtain different link travel times with the same cumulative flows. In this example, the maximum gap and average gap between the SF-type and LI-type link travel times are 2.50 seconds (less than $\frac{1}{2} \delta=5$ seconds) and 1.34 seconds, respectively. The maximum and average gaps between the SF-type and MLI-type link travel times are 2.14 seconds (less than $\frac{1}{2} \delta$ ) and 1.29 seconds, respectively. The maximum and average gaps between the LI-type and MLI-type link travel times are 0.57 seconds and 0.25 seconds, respectively. This result shows that the link travel times estimated by the LI-type and MLI-type travel time functions are very close.

From Table 3, we can observe that the cumulative outflow parameters estimated by the two types of models are essentially different. The result confirms that the LI-type cumulative outflow parameters are always greater than or equal to the MLI-type parameters.

To illustrate the monotonicity of each type of link travel time function, we directly calculated the Jacobian matrices of the SF-type and MLI-type link travel time functions (see Appendices B and E respectively), and calculated the Jacobian matrix of the LI-type link travel time function by numerical 
approximation. The inflow belonging to a particular interval was perturbed by a small amount and the LTM was applied to generate cumulative flows. Then the proposed LI-type formulation was employed to evaluate the travel times of all intervals with inflows. The differences of these values from the corresponding original link travel times divided by the perturbed inflow generate one column of the Jacobian matrix. The process was repeated for all the intervals to produce the full Jacobian matrix. The Jacobian matrices of the three type of link travel time functions are given as follows:

$$
\begin{aligned}
\nabla \mathbf{t}_{a}\left(\mathbf{y}_{a}\right) & =\left(\begin{array}{cccccc}
0.864 & 0 & 0 & 0 & 0 & 0 \\
0 & 0 & 0 & 0 & 0 & 0 \\
0 & 0 & 0.700 & 0 & 0 & 0 \\
0 & 0 & 1.667 & 1.042 & 0 & 0 \\
0 & 0 & 0 & 0 & 0 & 0 \\
0 & 0 & 0 & 0 & 0 & 0
\end{array}\right), \\
\nabla \hat{\mathbf{t}}_{a}\left(\mathbf{y}_{a}\right)= & \left(\begin{array}{ccccccc}
0.738 & -0.089 & 0 & 0 & 0 & 0 \\
0.600 & -0.400 & 0 & 0 & 0 & 0 \\
0 & 0 & 0.714 & 0 & 0 & 0 \\
0 & 0 & 1.437 & 0.722 & -0.012 & -0.012 \\
0 & 0 & 1.111 & 1.111 & 0.278 & -0.555 \\
0 & 0 & 0.417 & 0.417 & 0.417 & -0.417
\end{array}\right),
\end{aligned}
$$

and

$$
\nabla \overline{\mathbf{t}}_{a}\left(\mathbf{y}_{a}\right)=\left(\begin{array}{cccccc}
0.714 & 0 & 0 & 0 & 0 & 0 \\
0.714 & 0 & 0 & 0 & 0 & 0 \\
0 & 0 & 0.714 & 0 & 0 & 0 \\
0 & 0 & 1.429 & 0.714 & 0 & 0 \\
0 & 0 & 1.429 & 1.429 & 0.714 & 0 \\
0 & 0 & 0.714 & 0.714 & 0.714 & 0
\end{array}\right) .
$$

The results illustrate that Jacobian matrices of the SF-type and MLI-type link travel time functions are positive semidefinite and the two types of link travel time functions are monotone. Since $U_{a}(2)=$ $V_{a}(2+3)=12$ vehicles, and $m_{2} \neq n_{2}$, the link is not under the congestion condition. The Jacobian matrices presented in Eqs. (40) and (42) are lower triangular matrices. This result confirms that the SF-type and MLI-type link travel time functions satisfy causality.

The Jacobian matrix of the LI-type travel time function given in Eq. (41) indicates that this link travel time function is not a lower triangular matrix and hence does not satisfy causality. Therefore, some unrealistic results will be obtained from this type of travel time function. For example, if some more flows enter the link during intervals 2 and 6 , the link travel times of some intervals will decrease.

Example 3. With given inflows and outflows and under the link congestion condition. 
Compared with Example 2, three and two more vehicles are appended to enter link $a$ during interval 2 and interval 6, respectively. That is, the inflow is $\mathbf{y}_{a}=(9,6,10,12,2,5)^{\mathrm{T}}$. The other input parameters are the same as those in Example 2. In this example, the traffic flow is under the link congestion condition, because $U_{a}(k)<V_{a}(k+3)$ and $m_{k}=n_{k}$ are satisfied for all $k \leq 6$. The Jacobian matrices of the SF-type and MLI-type link travel time functions are given as follows:

$$
\nabla \mathbf{t}_{a}\left(\mathbf{y}_{a}\right)=\left(\begin{array}{cccccc}
0.864 & 0 & 0 & 0 & 0 & 0 \\
1.667 & 1.389 & 0 & 0 & 0 & 0 \\
1.000 & 1.000 & 0.700 & 0 & 0 & 0 \\
1.667 & 1.667 & 1.667 & 0.903 & 0 & 0 \\
0 & 0 & 0 & 0 & 0 & 0 \\
2.000 & 2.000 & 2.000 & 2.000 & 2.000 & 1.200
\end{array}\right),
$$

and

$$
\nabla \overline{\mathbf{t}}_{a}\left(\mathbf{y}_{a}\right)=\left(\begin{array}{cccccc}
0.714 & 0 & 0 & 0 & 0 & 0 \\
1.429 & 0.714 & 0 & 0 & 0 & 0 \\
1.429 & 1.429 & 0.714 & 0 & 0 & 0 \\
1.429 & 1.429 & 1.429 & 0.714 & 0 & 0 \\
1.429 & 1.429 & 1.429 & 1.429 & 0.714 & 0 \\
1.429 & 1.429 & 1.429 & 1.429 & 1.429 & 0.714
\end{array}\right) .
$$

The matrix $\nabla \mathbf{t}_{a}\left(\mathbf{y}_{a}\right)$ in Eq. (43) is positive semidefinite. This result implies that the SF-type link travel time function may not be strictly monotone under the congestion condition. In particular, for the interval $k=5$, we have $n_{k}=n_{k-1}$ intervals and $t_{a}(k)=\left(n_{k}-k\right) \delta$. By Eq. (51) in Appendix B, $\partial t_{a}(k) / \partial y_{a}(h)=0$ for all $h \in[1,6]$. Therefore, the values in the fifth row in Eq. (43) are equal to zero. Unlike $\nabla \mathbf{t}_{a}\left(\mathbf{y}_{a}\right)$, the matrix $\nabla \overline{\mathbf{t}}_{a}\left(\mathbf{y}_{a}\right)$ in Eq. (44) is positive definite. This implies that the MLI-type link travel time function is strictly monotone, which is consistent with Proposition 20.

Example 4. With a plateau shaped inflow profile.

In this example, we assume the link is empty initially. The input parameters for the LTM are given as follows:

- Jam density: 133 vehicles/km (i.e., 7.5m for every vehicle);

- Free-flow speed: $72 \mathrm{~km} / \mathrm{h}$ (i.e., $20 \mathrm{~m} / \mathrm{s}$ );

- Flow capacity: 36 veh/min;

- Link length: 2400m, and;

- Free flow travel time: 2 minutes. 
Following Carey and Ge (2005), we employ the following inflow function in this example:

$$
u_{a}(t)= \begin{cases}32 \sin \pi t / 10, & \text { if } 0 \leq t \leq 5, \\ 32, & \text { if } 5<t<10 \\ 20+12 \sin ^{5}(\pi(t+4) / 28), & \text { if } 10 \leq t \leq 20\end{cases}
$$

where the unit for the inflow is veh/min. We set a constant outflow capacity of $25.2 \mathrm{veh} / \mathrm{min}$. Again, the LTM was employed to generate cumulative flows. Then we applied the three types of models to these inflows and computed the link travel times and their mean errors over the study period under different interval lengths: $\delta=1,5,10,20,30,40,60$ seconds.

The link travel time results are shown in Fig. 7. As shown in this figure, the profiles of link travel times almost coincide with each other. This observation is consistent with Eqs. (33) and (34). The mean errors corresponding to each type of travel time models are also presented in Table 4 . This table shows that for a given value of $\delta$, the LI-type and MLI-type of link travel time models have a higher accuracy than the SF-type model in terms of all three error measures, but with a slight advantage for the MLI-type model over the LI-type model. We can also observe that MAE and MPE will uniformly increase for every type of link travel time models when we increase the value of $\delta$. This result indicates that shortening interval length can improve the accuracy of travel time models. However, the IRE of the SF-type model does not decrease but fluctuates around a constant value (e.g., about $3.18 \%$ in this example) even when a small value of $\delta$ is used. This result indicates that the total absolute calculation error cannot be eliminated by shortening interval length if we use the SF-type travel time model. The results in Table 4 also show that the IREs of the LI-type and MLI-type models are lower than the IRE of the SF-type model. Moreover, the IREs of the LI-type and MLI-type models can be effectively reduced by using a smaller value of $\delta$.

\section{Conclusion}

This paper developed three discretized travel time models based on cumulative flows which can be usually generated by DNL models. The first model, namely the SF-type model, assumes that the profiles of link cumulative inflows and outflows are piecewise linearized by step functions whereas the other two models, namely, LI-type and MLI-type models, assume that the profiles are piecewise linearized by linear interpolations. Their model properties such as (S)FIFO, causality, continuity and monotonicity were analyzed and elaborately discussed. Based on the analysis, we find that all the three models satisfy Link FIFO and continuity, no matter how large is the time step used for discretization. The SF-type model has a simpler calculation and ensures causality and monotonicity, while the LI-type model may potentially have a higher accuracy but also may disobey causality, and monotonicity. As an extended model of the LI-type model, the MLI-type model maintains both the accuracy and desirable properties such as ensuring strong FIFO, causality and monotonicity. The theoretical analysis demonstrates that 
the difference between the SF-type and (M)LI-type link travel times is less than 0.5 interval. This result indicates that if the interval length is small enough, these models will converge to the same solution.

In our numerical experiments, the first two examples were applied to demonstrate the properties of these link travel time models. The results show that the LI-type link travel time may disobey causality, which implies vehicles that enter a link later may influence vehicles that enter the link earlier. Compared with the SF-type model, the MLI-type model has more desirable properties: satisfying SFIFO and may be strictly monotone. The last example was employed to illustrate the accuracy of the models. The results indicate that the MLI-type model has the highest accuracy compared with the other two models.

Since both cumulative route and link flow curves have the monotonicity property, the SF-type and LItype link travel time models can be directly extended to calculate route travel times. Moreover, link FIFO implies route FIFO (Wu et al., 1998), and thus the extended route travel time models will satisfy route FIFO. We also can prove that the SF-type route travel time model satisfies causality, but the LI-type model does not. Unfortunately, it is very difficult to observe monotonicity of route travel time functions. Compared with the SF-type and LI-type models, the MLI-type model has more desirable properties (i.e., the travel time satisfies Link SFIFO, and is strictly monotone under the link congestion condition) and obtains travel times with a higher accuracy. However, the MLI-type model cannot be directly extended to route travel time. The calculation of the MLI-type link travel time relies on link outflow capacity, and we do not have outflow capacity corresponding to a route easily. Therefore, the MLI-type route travel time model should be improved in the future.

\section{Acknowledgements}

The authors would like to thank Prof. Haijun Huang, Prof. Malachy Carey, and the anonymous referees for their useful comments on this paper. This work is jointly supported by the National Basic Research Program of China (2006CB705500) and the National Natural Science Foundation of China (70631001).

\section{Appendix A}

Substituting Eq. (11) into Eq. (15) and rearranging the resulting expression, we have:

$$
\begin{aligned}
T_{a}(k) / \delta & =\sum_{l=1}^{\underline{K}}\left[Y_{a k}(l)-Y_{a k}(l-1)\right](l-k) \\
& =\sum_{l=1}^{\underline{K}} l\left[Y_{a k}(l)-Y_{a k}(l-1)\right]-k \sum_{l=1}^{\underline{K}}\left[Y_{a k}(l)-Y_{a k}(l-1)\right] \\
& =\sum_{l=1}^{\underline{K}} l Y_{a k}(l)-\sum_{l=1}^{\underline{K}} l Y_{a k}(l-1)-k Y_{a k}(\underline{K})+k Y_{a k}(0) \\
& =\sum_{l=1}^{\underline{K}} l Y_{a k}(l)-Y_{a k}(0)-\sum_{l=2}^{\underline{K}} l Y_{a k}(l-1)-k Y_{a k}(\underline{K})+k Y_{a k}(0) .
\end{aligned}
$$


By definition, the second and the last terms in Eq. (46) are equal to zero and $Y_{a k}(\underline{K})=y_{a}(k)$. Hence, Eq. (46) can be rewritten as follows:

$$
\begin{aligned}
T_{a}(k) / \delta & =\sum_{l=1}^{\underline{K}} l Y_{a k}(l)-\sum_{l=2}^{\underline{K}} l Y_{a k}(l-1)-k y_{a}(k) \\
& =\sum_{l=1}^{\underline{K}} l Y_{a k}(l)-\sum_{l=2}^{\underline{K}}(l-1) Y_{a k}(l-1)-\sum_{l=2}^{\underline{K}} Y_{a k}(l-1)-k y_{a}(k) \\
& =\sum_{l=1}^{\underline{K}} l Y_{a k}(l)-\sum_{l=1}^{\underline{K}-1} l Y_{a k}(l)-\sum_{l=1}^{\underline{K}-1} Y_{a k}(l)-k y_{a}(k) \\
& =\underline{K} y_{a}(k)-\sum_{l=1}^{\underline{K}-1} Y_{a k}(l)-k Y_{a k} \\
& =\underline{K}-k) y_{a}(k)-\sum_{l=1}^{\underline{K}-1} Y_{a k}(l) \\
& =(\underline{K}-k) y_{a}(k)-\sum_{l=1}^{n_{k-1}} Y_{a k}(l)-\sum_{l=n_{k-1}}^{n_{k}-1} Y_{a k}(l)-\sum_{l=n_{k}}^{\underline{K}-1} Y_{a k}(l) .
\end{aligned}
$$

Substituting Eqs. (8) and (14) into (47), we have:

$$
\begin{aligned}
T_{a}(k) / \delta & =(\underline{K}-k) y_{a}(k)-0-\sum_{l=n_{k-1}}^{n_{k}-1}\left[V_{a}(l)-U_{a}(k-1)\right]-\sum_{l=n_{k}}^{\underline{K}-1} y_{a}(k) \\
& =(\underline{K}-k) y_{a}(k)-\sum_{l=n_{k-1}}^{n_{k}-1}\left[V_{a}(l)-U_{a}(k-1)\right]-\left(\underline{K}-1-\left(n_{k}-1\right)\right) y_{a}(k) \\
& =\left(n_{k}-k\right) y_{a}(k)-\sum_{l=n_{k-1}}^{n_{k}-1}\left[V_{a}(l)-U_{a}(k-1)\right] .
\end{aligned}
$$

Therefore, we have:

$$
T_{a}(k)=\left(n_{k}-k\right) \delta y_{a}(k)-\sum_{l=n_{k-1}}^{n_{k}-1} \delta\left[V_{a}(l)-U_{a}(k-1)\right] .
$$

\section{Appendix B}

Below is the proof of Proposition 9.

Proof. We consider the inflow pattern $\mathbf{y}_{a}=\left(y_{a}(1), y_{a}(2), \cdots, y_{a}(k), y_{a}(k+1), \cdots\right)^{\mathrm{T}}$ and its perturbation at interval $h$ indicated as $\mathbf{y}_{a}^{\prime}=\left(y_{a}(1), y_{a}(2), \cdots, y_{a}(h)+\triangle, y_{a}(h+1), \cdots\right)^{\mathrm{T}}$, where $\triangle$ is arbitrary but satisfies $y_{a}(h)+\triangle \geq 0$. The variables corresponding to the inflow pattern $\mathbf{y}_{a}$ are added apostrophe to represent the variables corresponding to the inflow pattern $\mathbf{y}_{a}^{\prime}$.

To prove that the link travel time function is differentiable, we should prove that the following limits exist and is satisfied:

$$
\lim _{\Delta \rightarrow 0} \frac{t_{a}^{\prime}(k)-t_{a}(k)}{y_{a}^{\prime}(h)-y_{a}(h)}=\frac{\partial t_{a}(k)}{\partial y_{a}(h)}
$$


Proposition 3 indicates that $U_{a}(l)>V_{a}\left(m_{l}-1\right)$ for all $l \in K_{d}$, and this proposition assumes that $S_{a}\left(m_{l}\right)>U_{a}(l)-V_{a}\left(m_{l}-1\right)$ for all $l \in K_{d}$. We let:

$$
\psi=\min _{l}\left\{U_{a}(l)-V_{a}\left(m_{l}-1\right), S_{a}\left(m_{l}\right)-U_{a}(l)+V_{a}\left(m_{l}-1\right)\right\}>0 .
$$

and let $\Delta \in(-\psi, \psi)$, then $n_{l}^{\prime}=n_{l}$ is satisfied for all $l \in K_{d}$.

There are three cases:

Case I: $h<k$.

A small perturbation of inflow at interval $h$ does not always affect the link travel time at interval $k$. If $\exists l \in[h, k]$ such that $n_{l}=m_{l}+1$, then we have: $t_{a}^{\prime}(k)=t_{a}(k)$ and $\lim _{\Delta \rightarrow 0}\left[t_{a}^{\prime}(k)-t_{a}(k)\right] /\left[y_{a}^{\prime}(h)-y_{a}(h)\right]=$ 0 ; else if $n_{k}=n_{k-1}$, we also have: $t_{a}^{\prime}(k)=t_{a}(k)$ and $\lim _{\Delta \rightarrow 0}\left[t_{a}^{\prime}(k)-t_{a}(k)\right] /\left[y_{a}^{\prime}(h)-y_{a}(h)\right]=0$; Otherwise,

$$
y_{a k}^{\prime}(l)= \begin{cases}y_{a k}\left(n_{k-1}\right)-\Delta, & \text { if } l=n_{k-1}, \\ y_{a k}\left(n_{k}\right)+\Delta, & \text { if } l=n_{k}, \\ y_{a k}(l), & \text { otherwise. }\end{cases}
$$

Therefore,

$$
T_{a}^{\prime}(k)=\sum_{l=1}^{\underline{K}} y_{a k}^{\prime}(l)(l-k) \delta=T_{a}(k)-\Delta\left(n_{k-1}-k\right) \delta+\Delta\left(n_{k}-k\right) \delta
$$

and

$$
t_{a}^{\prime}(k)=T_{a}^{\prime}(k) / y_{a}^{\prime}(k)=\left[T_{a}(k)-\Delta\left(n_{k-1}-k\right) \delta+\Delta\left(n_{k}-k\right) \delta\right] / y_{a}^{\prime}(k) .
$$

We have:

$$
\begin{aligned}
\lim _{\Delta \rightarrow 0} \frac{t_{a}^{\prime}(k)-t_{a}(k)}{y_{a}^{\prime}(h)-y_{a}(h)} & =\lim _{\Delta \rightarrow 0} \frac{\left[T_{a}(k)-\Delta\left(n_{k-1}-k\right) \delta+\Delta\left(n_{k}-k\right) \delta\right] / y_{a}^{\prime}(k)-T_{a}(k) / y_{a}(k)}{\Delta} \\
& =\left(n_{k}-n_{k-1}\right) \delta / y_{a}(k) .
\end{aligned}
$$

Case II: $h=k$.

If $n_{k}=m_{k}+1$ is satisfied, then we have: $t_{a}^{\prime}(k)=t_{a}(k)$ and $\lim _{\Delta \rightarrow 0}\left[t_{a}^{\prime}(k)-t_{a}(k)\right] /\left[y_{a}^{\prime}(h)-y_{a}(h)\right]=0$; otherwise,

$$
y_{a k}^{\prime}(l)= \begin{cases}y_{a k}\left(n_{k}\right)+\Delta, & \text { if } l=n_{k} \\ y_{a k}(l), & \text { otherwise. }\end{cases}
$$

Therefore,

$$
T_{a}^{\prime}(k)=\sum_{l=1}^{\underline{K}} y_{a k}^{\prime}(l)(l-k) \delta=T_{a}(k)+\Delta\left(n_{k}-k\right) \delta,
$$

and

$$
t_{a}^{\prime}(k)=T_{a}^{\prime}(k) / y_{a}^{\prime}(k)=\left[T_{a}(k)+\Delta\left(n_{k}-k\right) \delta\right] /\left[y_{a}(k)+\Delta\right]
$$


We have:

$$
\begin{aligned}
\lim _{\Delta \rightarrow 0} \frac{t_{a}^{\prime}(k)-t_{a}(k)}{y_{a}^{\prime}(h)-y_{a}(h)} & =\lim _{\Delta \rightarrow 0} \frac{\left[T_{a}(k)+\Delta\left(n_{k}-k\right) \delta\right] /\left[y_{a}(k)+\Delta\right]-T_{a}(k) / y_{a}(k)}{\Delta} \\
& =\lim _{\Delta \rightarrow 0} T_{a}(k) \frac{1 /\left[y_{a}(k)+\Delta\right]-1 / y_{a}(k)}{\Delta}+\lim _{\Delta \rightarrow 0} \frac{\left(n_{k}-k\right) \delta}{y_{a}(k)+\Delta} \\
& =-\frac{T_{a}(k)}{\left[y_{a}(k)\right]^{2}}+\frac{\left(n_{k}-k\right) \delta}{y_{a}(k)} \\
& =\frac{\left(n_{k}-k\right) \delta-t_{a}(k)}{y_{a}(k)}
\end{aligned}
$$

$t_{a}(k)$ in Eq. (17) implies $n_{k}-k \geq t_{a}(k)$. Therefore, we have:

$$
\lim _{\Delta \rightarrow 0} \frac{t_{a}^{\prime}(k)-t_{a}(k)}{y_{a}^{\prime}(h)-y_{a}(h)} \geq 0
$$

Case III: $h>k$.

Since the dynamic link travel time calculated by Eq.(17) satisfies causality (see Proposition 5), we have $t_{a}^{\prime}(k)=t_{a}(k)$ and

$$
\lim _{\Delta \rightarrow 0} \frac{t_{a}^{\prime}(k)-t_{a}(k)}{y_{a}^{\prime}(h)-y_{a}(h)}=0 .
$$

The results of the above three cases can be summarized as follows:

$$
\frac{\partial t_{a}(k)}{\partial y_{a}(h)}=\lim _{\Delta \rightarrow 0} \frac{t_{a}^{\prime}(k)-t_{a}(k)}{y_{a}^{\prime}(h)-y_{a}(h)}= \begin{cases}\left(n_{k}-n_{k-1}\right) \delta / y_{a}(k), & \text { if } h<k, m_{l}=n_{l} \text { for all } l \in[h, k], \\ {\left[\left(n_{k}-k\right) \delta-t_{a}(k)\right] / y_{a}(k),} & \text { if } h=k, m_{k}=n_{k}, \\ 0, & \text { otherwise. }\end{cases}
$$

This completes the proof.

\section{Appendix C}

Below is the proof of Proposition 10.

Proof. Proposition 3 shows that $U_{a}(k)-V_{a}\left(m_{k}-1\right) \leq S_{a}\left(m_{k}\right)$ is satisfied for all $k \in K_{d}$. We firstly consider the case that $U_{a}(k)-V_{a}\left(m_{k}-1\right)<S_{a}\left(m_{k}\right), \forall k \in K_{d}$. Under this condition, Proposition 9 indicates that the function $\mathbf{t}_{a}\left(\mathbf{y}_{a}\right)$ is differentiable. Equation (51) indicates that the Jacobian matrix $\nabla \mathbf{t}_{a}\left(\mathbf{y}_{a}\right)$ is a lower triangular matrix and the diagonal entries of the matrix are non-negative. Thus, $\nabla \mathbf{t}_{a}\left(\mathbf{y}_{a}\right)$ is positive semidefinite. According to Lemma 1, the link travel time function $\mathbf{t}_{a}\left(\mathbf{y}_{a}\right)$ is monotone if $U_{a}(k)-V_{a}\left(m_{k}-1\right)<S_{a}\left(m_{k}\right), \forall k \in K_{d}$.

The definition domain of $\mathbf{t}_{a}\left(\mathbf{y}_{a}\right)$ can be divided into a group of continuous subdomains by the equations $U_{a}(k)-V_{a}\left(m_{k}-1\right)=S_{a}\left(m_{k}\right), \forall k \in K_{d}$. If the boundary of each subdomain is not considered (i.e., $\left.U_{a}(k)-V_{a}\left(m_{k}-1\right)<S_{a}\left(m_{k}\right), \forall k \in K_{d}\right), \mathbf{t}_{a}\left(\mathbf{y}_{a}\right)$ is monotone on each subdomain. For each subdomain, $\mathbf{t}_{a}\left(\mathbf{y}_{a}\right)$ is also monotone on the boundary. Therefore, $\mathbf{t}_{a}\left(\mathbf{y}_{a}\right)$ is monotone on all subdomains. This implies that $\mathbf{t}_{a}\left(\mathbf{y}_{a}\right)$ is monotone on the definition domain (i.e., $\mathbf{y}_{a}>0$ ). 


\section{Appendix D}

Below is the proof of Proposition 11.

Proof. Similar to the proof of Proposition 5, we aim to prove that $\hat{t}_{a}(k+1)-\hat{t}_{a}(k)+\delta>0$ is satisfied. $\hat{T}_{a}(k)$ in Eq. (29) can be reformulated as follows:

$\hat{T}_{a}(k)=t_{a}(k) y_{a}(k)+\frac{1}{2} \delta\left[\mu_{k-1} y_{a k}\left(n_{k-1}\right)+\left(\mu_{k}-1\right) y_{a k}\left(n_{k}\right)\right]=T_{a}(k)+\frac{1}{2} \delta\left[\mu_{k-1} y_{a k}\left(n_{k-1}\right)+\left(\mu_{k}-1\right) y_{a k}\left(n_{k}\right)\right]$, where the first term in the second equality is obtained by the definition of $T_{a}(k)$ in Eq (17). Substituting $y_{a}(k)=Y_{a k}\left(n_{k}\right)$, Eq. (14) and Eq. (16) into the above, we have:

$$
\begin{aligned}
\frac{\hat{T}_{a}(k)}{\delta} & =\left(n_{k}-k\right) Y_{a k}\left(n_{k}\right)-\sum_{l=n_{k-1}}^{n_{k}-1} Y_{a k}(l)+\frac{1}{2} \mu_{k-1} Y_{a k}\left(n_{k-1}\right)+\frac{1}{2}\left(\mu_{k}-1\right)\left[Y_{a k}\left(n_{k}\right)-Y_{a k}\left(n_{k}-1\right)\right] \\
& =\left[n_{k}-k+\frac{1}{2}\left(\mu_{k}-1\right)\right] Y_{a k}\left(n_{k}\right) \\
& -\left\{\frac{1}{2} \sum_{l=n_{k-1}+1}^{n_{k}-1} Y_{a k}(l)+\frac{1}{2}\left(1-\mu_{k-1}\right) Y_{a k}\left(n_{k-1}\right)+\frac{1}{2} \sum_{l=n_{k-1}}^{n_{k}-2} Y_{a k}(l)+\frac{1}{2} \mu_{k} Y_{a k}\left(n_{k}-1\right)\right\} .
\end{aligned}
$$

By Eq. (29) and (52), we get:

$$
\begin{aligned}
\hat{t}_{a}(k) / \delta & =\hat{T}_{a}(k) / \delta Y_{a k}\left(n_{k}\right) \\
& =\left[n_{k}-k+\frac{1}{2}\left(\mu_{k}-1\right)\right] \\
& -\left\{\frac{1}{2} \sum_{l=n_{k-1}+1}^{n_{k}-1} Y_{a k}(l)+\frac{1}{2}\left(1-\mu_{k-1}\right) Y_{a k}\left(n_{k-1}\right)+\frac{1}{2} \sum_{l=n_{k-1}}^{n_{k}-2} Y_{a k}(l)+\frac{1}{2} \mu_{k} Y_{a k}\left(n_{k}-1\right)\right\} / Y_{a k}\left(n_{k}\right) .
\end{aligned}
$$

Since $0 \leq \mu_{k} \leq 1$ for all $k$ and $Y_{a k}(l) \geq 0$ for all $l$, the brace term

$$
\frac{1}{2} \sum_{l=n_{k-1}+1}^{n_{k}-1} Y_{a k}(l)+\frac{1}{2}\left(1-\mu_{k-1}\right) Y_{a k}\left(n_{k-1}\right)+\frac{1}{2} \sum_{l=n_{k-1}}^{n_{k}-2} Y_{a k}(l)+\frac{1}{2} \mu_{k} Y_{a k}\left(n_{k}-1\right) \geq 0 .
$$

Moreover, because $Y_{a k}(l) \leq Y_{a k}\left(n_{k}\right)=y_{a}(k), Y_{a k}(l) \geq 0$, and $Y_{a k}\left(n_{k}-1\right) \leq Y_{a k}\left(n_{k}\right)$ for all $k$ and $l$, the brace term

$$
\begin{aligned}
& \frac{1}{2} \sum_{l=n_{k-1}+1}^{n_{k}-1} Y_{a k}(l)+\frac{1}{2}\left(1-\mu_{k-1}\right) Y_{a k}\left(n_{k-1}\right)+\frac{1}{2} \sum_{l=n_{k-1}}^{n_{k}-2} Y_{a k}(l)+\frac{1}{2} \mu_{k} Y_{a k}\left(n_{k}-1\right) \\
& \leq \frac{1}{2} \sum_{l=n_{k-1}+1}^{n_{k}-1} Y_{a k}\left(n_{k}\right)+\frac{1}{2}\left(1-\mu_{k-1}\right) Y_{a k}\left(n_{k}\right)+\frac{1}{2} \sum_{l=n_{k-1}}^{n_{k}-2} Y_{a k}\left(n_{k}\right)+\frac{1}{2} \mu_{k} Y_{a k}\left(n_{k}\right) \\
& =\left[n_{k}-n_{k-1}-\frac{1}{2} \mu_{k-1}+\frac{1}{2}\left(\mu_{k}-1\right)\right] Y_{a k}\left(n_{k}\right) .
\end{aligned}
$$

If $n_{k}>n_{k-1}$, Eqs. (54) and (55) combined with Eq. (53) imply

$$
\left(n_{k-1}-k+\frac{1}{2} \mu_{k-1}\right) \delta \leq \hat{t}_{a}(k) \leq\left[n_{k}-k+\frac{1}{2}\left(\mu_{k}-1\right)\right] \delta .
$$


If $n_{k-1}=n_{k}$, by Eqs. (27) and (29), we have:

$$
\hat{t}_{a}(k)=\left[\left(n_{k}-k\right)+\frac{1}{2}\left(\mu_{k-1}+\mu_{k}-1\right)\right] \delta .
$$

Eqs. (56) and (57) will be used for the following four cases:

Case I: $n_{k-1}=n_{k}=n_{k+1}$.

$$
\begin{aligned}
& \hat{t}_{a}(k+1)-\hat{t}_{a}(k)+\delta \\
& =\left[\left(n_{k+1}-k-1\right)+\frac{1}{2}\left(\mu_{k}+\mu_{k+1}-1\right)\right] \delta-\left[\left(n_{k}-k\right)+\frac{1}{2}\left(\mu_{k-1}+\mu_{k}-1\right)\right] \delta+\delta \\
& =\frac{1}{2}\left(\mu_{k+1}-\mu_{k-1}\right) \delta .
\end{aligned}
$$

In this case, Theorem 1 implies $\mu_{k+1}>\mu_{k}>\mu_{k-1}$, and $\hat{t}_{a}(k+1)-\hat{t}_{a}(k)+\delta>0$ is satisfied.

Case II: $n_{k-1}<n_{k}=n_{k+1}$.

$$
\begin{aligned}
& \hat{t}_{a}(k+1)-\hat{t}_{a}(k)+\delta \\
& \geq\left[\left(n_{k+1}-k-1\right)+\frac{1}{2}\left(\mu_{k}+\mu_{k+1}-1\right)\right] \delta-\left[n_{k}-k+\frac{1}{2}\left(\mu_{k}-1\right)\right] \delta+\delta \\
& =\frac{1}{2} \mu_{k+1} \delta .
\end{aligned}
$$

In this case, Theorem 1 implies $\mu_{k+1}>0$, and $\hat{t}_{a}(k+1)-\hat{t}_{a}(k)+\delta>0$ is satisfied.

Case III: $n_{k-1}=n_{k}<n_{k+1}$.

$$
\begin{aligned}
& \hat{t}_{a}(k+1)-\hat{t}_{a}(k)+\delta \\
& \geq\left[\left(n_{k}-k-1+\frac{1}{2} \mu_{k}\right)\right] \delta-\left[\left(n_{k}-k\right)+\frac{1}{2}\left(\mu_{k-1}+\mu_{k}-1\right)\right] \delta+\delta \\
& =\frac{1}{2}\left(1-\mu_{k-1}\right) \delta .
\end{aligned}
$$

In this case, Theorem 1 implies $\mu_{k-1}<1$, and therefore $\frac{1}{2}\left(1-\mu_{k-1}\right)>0$. Thus, $\hat{t}_{a}(k+1)-\hat{t}_{a}(k)+\delta>$ 0 is satisfied.

Case IV: $n_{k-1}<n_{k}<n_{k+1}$.

$$
\begin{aligned}
& \hat{t}_{a}(k+1)-\hat{t}_{a}(k)+\delta \\
& \geq\left[\left(n_{k}-k-1+\frac{1}{2} \mu_{k}\right)\right] \delta-\left[n_{k}-k+\frac{1}{2}\left(\mu_{k}-1\right)\right] \delta+\delta \\
& =\frac{1}{2} \delta .
\end{aligned}
$$

The above four cases demonstrate $\hat{t}_{a}(k+1)-\hat{t}_{a}(k)+\delta>0$. This completes the proof. 


\section{Appendix E}

Below is the proof of Proposition 17.

Proof. Similar to the proof of Proposition 9, we consider the inflow pattern $\mathbf{y}_{a}=\left(y_{a}(1), y_{a}(2), \cdots, y_{a}(k)\right.$, $\left.y_{a}(k+1), \cdots\right)^{\mathrm{T}}$ and its perturbation at interval $h$ indicated as $\mathbf{y}_{a}^{\prime}=\left(y_{a}(1), y_{a}(2), \cdots, y_{a}(h)+\triangle, y_{a}(h+\right.$ $1), \cdots)^{\mathrm{T}}$, where $\triangle$ is arbitrary but satisfies $y_{a}(h)+\triangle \geq 0$. The variables corresponding to the inflow pattern $\mathbf{y}_{a}$ are added apostrophe to represent the variables corresponding to inflow pattern $\mathbf{y}_{a}^{\prime}$.

To prove that the link travel time function is differentiable, we should prove that the following limits exist and is satisfied

$$
\lim _{\Delta \rightarrow 0} \frac{\bar{t}_{a}^{\prime}(k)-\bar{t}_{a}(k)}{y_{a}^{\prime}(h)-y_{a}(h)}=\frac{\partial \bar{t}_{a}(k)}{\partial y_{a}(h)} .
$$

We let $\Delta \in(-\psi, \psi)(\psi$ can be calculated by Eq. (50)), and three cases should be considered:

Case I: $h<k$.

A small perturbation of inflow at interval $h$ does not always affect the link travel time at interval $k$. If $\exists l \in[h, k)$ such that $n_{l}=m_{l}+1$, then we have $\bar{t}_{a}^{\prime}(k)=\bar{t}_{a}(k)$ and $\lim _{\Delta \rightarrow 0}\left[t_{a}^{\prime}(k)-t_{a}(k)\right] /\left[y_{a}^{\prime}(h)-\right.$ $\left.y_{a}(h)\right]=0$; else if $n_{k}=m_{k}+1$, we have: $\bar{\mu}_{k-1}^{\prime}=\bar{\mu}_{k-1}+\Delta / S_{a}\left(n_{k-1}\right), \bar{\mu}_{k}^{\prime}=\bar{\mu}_{k}, t_{a}^{\prime}(k)=t_{a}(k)$, $y_{a k}\left(n_{k-1}\right)=y_{a}(k), y_{a k}^{\prime}(l)=y_{a k}(l)$ for all $l \in K_{d}$. Therefore, $\bar{t}_{a}^{\prime}(k)$ can be calculated by Eq. (31):

$$
\bar{t}_{a}^{\prime}(k)=t_{a}(k)+\frac{1}{2} \delta\left[\bar{\mu}_{k-1}^{\prime} y_{a k}\left(n_{k-1}\right)+\left(\bar{\mu}_{k}-1\right) y_{a k}\left(n_{k}\right)\right] / y_{a}(k) .
$$

Then, we have:

$$
\lim _{\Delta \rightarrow 0} \frac{\bar{t}_{a}^{\prime}(k)-\bar{t}_{a}(k)}{y_{a}^{\prime}(h)-y_{a}(h)}=\frac{\delta}{2 S_{a}\left(n_{k-1}\right)} .
$$

If $n_{l} \neq m_{l}+1$ (i.e., $n_{l}=m_{l}$ ) for all $l \in[l, k]$, we have: $\bar{\mu}_{k-1}^{\prime}=\bar{\mu}_{k-1}+\Delta / S_{a}\left(n_{k-1}\right)$ and $\bar{\mu}_{k}^{\prime}=$ $\bar{\mu}_{k}+\Delta / S_{a}\left(n_{k}\right)$. Therefore, $\bar{t}_{a}^{\prime}(k)$ can be calculated by Eq. (31):

$$
\bar{t}_{a}^{\prime}(k)=t_{a}^{\prime}(k)+\frac{1}{2} \delta\left\{\bar{\mu}_{k-1}^{\prime}\left[y_{a k}\left(n_{k-1}\right)-\Delta\right]+\left(\bar{\mu}_{k}^{\prime}-1\right)\left[y_{a k}\left(n_{k}\right)+\Delta\right]\right\} / y_{a}(k) .
$$

Therefore, we have:

$$
\lim _{\Delta \rightarrow 0} \frac{\bar{t}_{a}^{\prime}(k)-\bar{t}_{a}(k)}{y_{a}^{\prime}(h)-y_{a}(h)}=\frac{\left[n_{k}-n_{k-1}+\bar{\mu}_{k}-\bar{\mu}_{k-1}\right] \delta}{y_{a}(k)} .
$$

Case II: $h=k$.

If $n_{k}=m_{k}+1$, then we have: $\bar{t}_{a}^{\prime}(k)=\bar{t}_{a}(k)$ and $\lim _{\Delta \rightarrow 0}\left[t_{a}^{\prime}(k)-t_{a}(k)\right] /\left[y_{a}^{\prime}(h)-y_{a}(h)\right]=0$. Otherwise, we have $\bar{\mu}_{k-1}^{\prime}=\bar{\mu}_{k-1}$ and $\bar{\mu}_{k}^{\prime}=\bar{\mu}_{k}+\Delta / S_{a}\left(n_{k}\right)$. Therefore, $\bar{t}_{a}^{\prime}(k)$ can be calculated by Eq. (26):

$$
\bar{t}_{a}^{\prime}(k)=t_{a}^{\prime}(k)+\frac{1}{2} \delta\left\{\bar{\mu}_{k-1} y_{a k}\left(n_{k-1}\right)+\left(\bar{\mu}_{k}^{\prime}-1\right)\left[y_{a k}\left(n_{k}\right)+\Delta\right]\right\} /\left[y_{a}(k)+\Delta\right] .
$$

Define $\bar{g}_{a}(k)=\bar{t}_{a}(k)-t_{a}(k)$ and $\bar{g}_{a}^{\prime}(k)=\bar{t}_{a}^{\prime}(k)-t_{a}^{\prime}(k)$. Then, by Eq.(31) we get:

$$
\bar{g}_{a}(k)=\frac{1}{2} \delta\left[\bar{\mu}_{k-1} y_{a k}\left(n_{k-1}\right)+\left(\bar{\mu}_{k}-1\right) y_{a k}\left(n_{k}\right)\right] / y_{a}(k),
$$


and

$$
\bar{g}_{a}^{\prime}(k)=\frac{1}{2} \delta\left\{\bar{\mu}_{k-1} y_{a k}\left(n_{k-1}\right)+\left(\bar{\mu}_{k}^{\prime}-1\right)\left[y_{a k}\left(n_{k}\right)+\Delta\right]\right\} /\left[y_{a}(k)+\Delta\right] .
$$

Consequently,

$$
\begin{aligned}
& \lim _{\Delta \rightarrow 0} \frac{\bar{t}_{a}^{\prime}(k)-\bar{t}_{a}(k)}{y_{a}^{\prime}(h)-y_{a}(h)} \\
& =\lim _{\Delta \rightarrow 0} \frac{\left[t_{a}^{\prime}(k)+\bar{g}_{a}^{\prime}(k)\right]-\left[t_{a}(k)+\bar{g}_{a}(k)\right]}{y_{a}^{\prime}(h)-y_{a}(h)} \\
& =\lim _{\Delta \rightarrow 0} \frac{t_{a}^{\prime}(k)-t_{a}(k)}{\Delta}+\lim _{\Delta \rightarrow 0} \frac{\bar{g}_{a}^{\prime}(k)-\bar{g}_{a}(k)}{\Delta} \\
& =\frac{\left(n_{k}-k\right) \delta-t_{a}(k)}{y_{a}(k)}+\lim _{\Delta \rightarrow 0} \frac{2\left\{y_{a}(k)\left[y_{a}(k)+\Delta\right] \bar{g}_{a}^{\prime}(k)-y_{a}(k)\left[y_{a}(k)+\Delta\right] \bar{g}_{a}(k)\right\} / \delta}{2 y_{a}(k)\left[y_{a}(k)+\Delta\right] \Delta / \delta} .
\end{aligned}
$$

The first term above is obtained using the result in Eq. (51). Since $\bar{\mu}_{k}=\left[U_{a}(k)-V_{a}\left(n_{k}-\right.\right.$ $1)] / S_{a}\left(n_{k}\right)=y_{a k}\left(n_{k}\right) / S_{a}\left(n_{k}\right)$, and $\frac{1}{2} \delta\left[\bar{\mu}_{k-1} y_{a k}\left(n_{k-1}\right)+\left(\bar{\mu}_{k}-1\right) y_{a k}\left(n_{k}\right)\right]=\left[\bar{t}_{a}(k)-t_{a}(k)\right] y_{a}(k)$ (see Eq. (31)), the numerator of the second term can be calculated as follows:

$$
\begin{aligned}
& 2\left\{y_{a}(k)\left[y_{a}(k)+\Delta\right] \bar{g}_{a}^{\prime}(k)-y_{a}(k)\left[y_{a}(k)+\Delta\right] \bar{g}_{a}(k)\right\} / \delta \\
& \left.=y_{a}(k)\left\{\bar{\mu}_{k-1} y_{a k}\left(n_{k-1}\right)+\left(\bar{\mu}_{k}^{\prime}-1\right)\left[y_{a k}\left(n_{k}\right)+\Delta\right]\right)\right\}-\left[y_{a}(k)+\Delta\right]\left[\bar{\mu}_{k-1} y_{a k}\left(n_{k-1}\right)+\left(\bar{\mu}_{k}-1\right) y_{a k}\left(n_{k}\right)\right] \\
& \left.=y_{a}(k)\left\{\bar{\mu}_{k-1} y_{a k}\left(n_{k-1}\right)+\left[\bar{\mu}_{k}+\frac{\Delta}{S_{a}\left(n_{k}\right)}-1\right]\left[y_{a k}\left(n_{k}\right)+\Delta\right]\right)\right\}-y_{a}(k)\left[\bar{\mu}_{k-1} y_{a k}\left(n_{k-1}\right)+\left(\bar{\mu}_{k}-1\right) y_{a k}\left(n_{k}\right)\right] \\
& -\left[\bar{\mu}_{k-1} y_{a k}\left(n_{k-1}\right)+\left(\bar{\mu}_{k}-1\right) y_{a k}\left(n_{k}\right)\right] \Delta \\
& =\frac{y_{a}(k) y_{a k}\left(n_{k}\right) \Delta}{S_{a}\left(n_{k}\right)}+y_{a}(k)\left(\bar{\mu}_{k}-1\right) \Delta+2 \frac{y_{a}(k) \Delta^{2}}{S_{a}\left(n_{k}\right)}-\left[\bar{\mu}_{k-1} y_{a k}\left(n_{k-1}\right)+\left(\bar{\mu}_{k}-1\right) y_{a k}\left(n_{k}\right)\right] \Delta \\
& =2 y_{a}(k) \bar{\mu}_{k} \Delta-y_{a}(k) \Delta+2 \frac{y_{a}(k) \Delta^{2}}{S_{a}\left(n_{k}\right)}-2\left[\bar{t}_{a}(k)-t_{a}(k)\right] y_{a}(k) \Delta / \delta .
\end{aligned}
$$

Thus,

$$
\lim _{\Delta \rightarrow 0} \frac{2\left\{y_{a}(k)\left[y_{a}(k)+\Delta\right] \bar{g}_{a}^{\prime}(k)-y_{a}(k)\left[y_{a}(k)+\Delta\right] \bar{g}_{a}(k)\right\} / \delta}{2 y_{a}(k)\left[y_{a}(k)+\Delta\right] \Delta / \delta}=\frac{\left(\mu_{k}-\frac{1}{2}\right) \delta-\left[\bar{t}_{a}(k)-t_{a}(k)\right]}{y_{a}(k)} .
$$

Therefore, we have:

$\lim _{\Delta \rightarrow 0} \frac{\bar{t}_{a}^{\prime}(k)-\bar{t}_{a}(k)}{y_{a}^{\prime}(h)-y_{a}(h)}=\frac{\left(n_{k}-k\right) \delta-t_{a}(k)}{y_{a}(k)}+\frac{\left(\bar{\mu}_{k}-\frac{1}{2}\right) \delta-\left[\bar{t}_{a}(k)-t_{a}(k)\right]}{y_{a}(k)}=\frac{\left(n_{k}-k+\bar{\mu}_{k}-\frac{1}{2}\right) \delta-\bar{t}_{a}(k)}{y_{a}(k)}$.

In addition,

$$
\begin{aligned}
& \frac{\left[n_{k}-k+\bar{\mu}_{k}-\frac{1}{2}\right] \delta-\bar{t}_{a}(k)}{y_{a}(k)} \\
& =\frac{\left[n_{k}-k+\bar{\mu}_{k}-\frac{1}{2}\right] \delta-\left\{t_{a}(k)+\frac{1}{2} \delta\left[\bar{\mu}_{k-1} y_{a k}\left(n_{k-1}\right)+\left(\bar{\mu}_{k}-1\right) y_{a k}\left(n_{k}\right)\right] / y_{a}(k)\right\}}{y_{a}(k)} \\
& =\frac{\frac{1}{2} \bar{\mu}_{k} Y_{a k}\left(n_{k}\right)+\sum_{l=n_{k-1}}^{n_{k}-1} Y_{a k}(l)-\frac{1}{2} \bar{\mu}_{k-1} Y_{a k}\left(n_{k-1}\right)-\frac{1}{2}\left(1-\bar{\mu}_{k}\right) Y_{a k}\left(n_{k}-1\right)}{y_{a}(k)^{2} / \delta} \\
& \geq \frac{\frac{1}{2} \bar{\mu}_{k} Y_{a k}\left(n_{k}\right)}{y_{a}(k)^{2} / \delta} \\
& =\frac{\bar{\mu}_{k} \delta}{2 y_{a}(k)} \\
& \geq 0 .
\end{aligned}
$$


Case III: $h>k$

Since the dynamic link travel time calculated by Eq. (31) satisfies causality (see Proposition 15), we have $\bar{t}_{a}^{\prime}(k)=\bar{t}_{a}(k)$ and

$$
\lim _{\Delta \rightarrow 0} \frac{\bar{t}_{a}^{\prime}(k)-\bar{t}_{a}(k)}{y_{a}^{\prime}(h)-y_{a}(h)}=0
$$

The results of upper three cases can be summarized as follows:

$$
\frac{\partial \bar{t}_{a}(k)}{\partial y_{a}(h)}= \begin{cases}\delta / 2 S_{a}\left(n_{k-1}\right), & \text { if } h<k, m_{k} \neq n_{k}, m_{l}=n_{l} \text { for all } l \in[h, k), \\ {\left[n_{k}-n_{k-1}+\bar{\mu}_{k}-\bar{\mu}_{k-1}\right] \delta / y_{a}(k),} & \text { if } h<k, m_{l}=n_{l} \text { for all } l \in[h, k], \\ {\left[\left(n_{k}-k+\bar{\mu}_{k}-1 / 2\right) \delta-\bar{t}_{a}(k)\right] / y_{a}(k),} & \text { if } h=k, m_{k}=n_{k}, \\ 0, & \text { otherwise. }\end{cases}
$$

This completes the proof.

\section{Appendix F}

Below is the proof of Proposition 20.

Proof. Similar to the proof of Proposition 10 in Appendix C, we also consider the case that $U_{a}(k)-$ $V_{a}\left(m_{k}-1\right)<S_{a}\left(m_{l}\right), \forall l \in K_{d}$. Under this condition, Proposition 17 indicates that the function $\overline{\mathbf{t}}_{a}\left(\mathbf{y}_{a}\right)$ is differentiable. Proposition 17 implies that $\bar{\mu}>0$ if link $a$ is under the congestion condition, and therefore Eq. (59) indicates that the Jacobian matrix $\nabla \overline{\mathbf{t}}_{a}\left(\mathbf{y}_{a}\right)$ is a lower triangular matrix and the diagonal entries of the matrix are positive. Thus, $\nabla \overline{\mathbf{t}}_{a}\left(\mathbf{y}_{a}\right)$ is positive definite. According to Lemma 1, the link travel time function $\overline{\mathbf{t}}_{a}\left(\mathbf{y}_{a}\right)$ is strictly monotone if $U_{a}(l)-V_{a}\left(m_{l}-1\right)<S_{a}\left(m_{l}\right), \forall l \in K_{d}$. Similar to the proof of Proposition 10, we can prove that $\overline{\mathbf{t}}_{a}\left(\mathbf{y}_{a}\right)$ is strictly monotone under the link congestion condition.

\section{References}

Astarita, V., 1996. A continuous time link model for dynamic network loading models based on travel time function. In Lesort J.B. (ed), Transportation and Traffic Theory, Pergamon Elservier, New York, 79-102.

Carey, M., Ge, Y.E., McCartney, M., 2003. A whole-link travel-time model with desirable properties. Transportation Science 37(1), 83-96.

Carey, M., Ge, Y.E., 2005. Convergence of a discretised travel-time model. Transportation Science 39(1), $25-38$.

Carey, M., Ge, Y.E., 2007. Retaining desirable properties in discretising a travel-time model. Transportation Research Part B 41(5), 540-553. 
Carey M., Srinivasan, A., 1993. Externalities, average and marginal costs, and tolls on congested networks with time-varying flows. Operations Research 41(1), 217-231.

Chen, H.K., 1999. Dynamic travel choice models: a variational inequality approach. Springer, Berlin, Germany, 27-28.

Daganzo, C.F., 1994. The cell transmission model: a simple dynamic representation of highway traffic. Transportation Research Part B 28 (4), 269-287.

Daganzo, C.F., 1995a. The cell transmission model, Part II: Network traffic. Transportation Research Part B 29(2), 79- 93.

Daganzo, C.F., 1995b. Properties of link travel time functions under dynamic loads. Transportation Research Part B 29(2), 95-98.

Friesz, T.L., Bernstein, D.H., Smith, T.E., Tobin, R.L., Wie, B.W., 1993. A variational inequality formulation of the dynamic network user equilibrium problem. Operations Research 41(1), 179-191.

Huang, H.J., Lam, W.H.K., 2002. Modeling and solving the dynamic user equilibrium route and departure time choice problem in network with queues. Transportation Research Part B 36(3), 253-273.

Kuwahara, M., Akamatsu, T., 2001. Dynamic user optimal assignment with physical queues for a manyto-many OD pattern. Transportation Research Part B 35(5), 461-479.

Lam, W.H.K., Huang, H.J., 1995. Dynamic user optimal traffic assignment model for many to one travel demand. Transportation Research Part B 29(4), 243-259.

Lian, A.P., Gao, Z.Y., Long, J.C., 2007. A dynamic user optimal assignment problem of link variables based on the cell transmission model. Acta Automatica Sinica 33(8), 852-859. (in Chinese)

Lighthill, M.H., Whitham, G.B., 1955. On kinematics wave: II A theory of traffic flow on long crowed roads. Proceedings of the Royal Society, London, Series A 229(1178), 317-345.

Lo, H.K., Szeto, W.Y., 2002. A cell-based variational inequality formulation of the dynamic user optimal assignment problem. Transportation Research Part B 36(5), 421-443.

Merchant, D.K., Nemhauser, G.L., 1978. A model and an algorithm for the dynamic traffic assignment problem. Transportation Science 12, 183-199.

Nagurney, A., 1993. Network economics: a variational inequality approach. Kluwer Academic Publishers. Norwell, Massachusetts, USA.

Newell, G.F., 1993. A simplified theory on kinematic wave in highway traffic, Part I: General theory; Part II: Queuing at freeway bottlenecks; Part III: Multi-destination flows, Transportation Research Part B 27(4), 281-314. 
Nie, X.J., 2003. The study of dynamic user-equilibrium traffic assignment. Ph.D. Thesis, University of California, Davis.

Nie, Y., Zhang, H.M., 2010. Solving the dynamic user optimal assignment problem considering queue spillback. Networks and Spatial Economics 10(1), 49 - 71.

Ran, B., Boyce, D.E., 1996. Modelling Dynamic Transportation Networks. Springer-Verlag, Heidelberg, Germany.

Richards, P.I., 1956. Shock waves on the highway. Operations Research 4(1), 42-51.

Shin, M.S., Ran B., He, R.R., 2000. Introducing platoon dispersion into analytical dynamic assignment process. Transportation Research Record 1733, 96-104.

Szeto, W.Y., 2008. The enhanced lagged cell transmission model for dynamic traffic assignment, Transportation Research Record 2085, 76-85.

Szeto, W.Y., Lo, H.K., 2004. A cell-based simultaneous route and departure time choice model with elastic demand. Transportation Research Part B 38(7), 593-612.

Szeto, W.Y., Lo, H.K., 2006. Dynamic traffic assignment: properties and extensions. Transportmetrica $2(1), 31-52$.

Wie, B.W., Tobin, R.L., 1998. Dynamic congestion pricing models for general traffic networks. Transportation Research Part B 32(5), 313-327.

Wu, J.H., Chen, Y., Florian, M., 1998. The continuous dynamic network loading problem: a mathematical formulation and solution method. Transportation Research Part B 32(3), 173-187.

Yperman, I., 2007. The link transmission model for dynamic network loading. Ph.D. Thesis, Katholieke Universiteit Leuven, Leuven, Belgium.

Ziliaskopoulos, A.K., 2000. A linear programming model for the single destination system optimum dynamic traffic assignment problem. Transportation Science 34(1), 37-49. 
Table 1: A comparison of the properties between various types of link travel time functions.

\begin{tabular}{llll}
\hline Property & SF-type & LI-type & MLI-type \\
\hline Link FIFO & Satisfy & Satisfy & Satisfy \\
Link SFIFO & Not satisfy & Satisfy & Satisfy \\
Causality & Satisfy & Not satisfy & Satisfy \\
Continuity & Satisfy & Satisfy & Satisfy \\
Monotone & Satisfy & Not satisfy & Satisfy \\
Strictly monotone & Not satisfy & Not satisfy & May satisfy \\
\hline
\end{tabular}

Table 2: The flows and cumulative flows in Example 2.

\begin{tabular}{lrrrrrrrrrr}
\hline$k$ (interval) & 1 & 2 & 3 & 4 & 5 & 6 & 7 & 8 & 9 & 10 \\
\hline$y_{a}(k)($ veh $)$ & 9 & 3 & 10 & 12 & 2 & 3 & - & - & - & - \\
$U_{a}(k)($ veh $)$ & 9 & 12 & 22 & 34 & 36 & 39 & 39 & 39 & 39 & 39 \\
$v_{a}(k) \delta($ veh $)$ & 0 & 0 & 0 & 7 & 5 & 7 & 7 & 7 & 6 & 0 \\
$V_{a}(k)($ veh $)$ & 0 & 0 & 0 & 7 & 12 & 19 & 26 & 33 & 39 & 39 \\
\hline
\end{tabular}

Table 3: The results related to various types of link travel times in Example 2.

\begin{tabular}{lrrrrrr}
\hline$k$ (interval) & 1 & 2 & 3 & 4 & 5 & 6 \\
\hline$m_{k}$ (interval) & 5 & 5 & 7 & 9 & 9 & 9 \\
$n_{k}$ (interval) & 5 & 6 & 7 & 9 & 9 & 10 \\
$\mu_{k}$ (interval) & $2 / 5$ & 0 & $3 / 7$ & $1 / 6$ & $3 / 6$ & 0 \\
$\bar{\mu}_{k}$ (interval) & $2 / 7$ & 0 & $3 / 7$ & $1 / 7$ & $3 / 7$ & 0 \\
$t_{a}(k)$ (sec) & 32.22 & 30.00 & 33.00 & 37.50 & 40.00 & 30.00 \\
$\hat{t}_{a}(k)$ (sec) & 31.56 & 32.00 & 32.14 & 37.87 & 38.33 & 32.50 \\
$\bar{t}_{a}(k)$ (sec) & 31.43 & 31.43 & 32.14 & 37.86 & 37.86 & 32.14 \\
\hline
\end{tabular}


Table 4: Errors of link travel time calculation in Example 4.

\begin{tabular}{llllllllll}
\hline & \multicolumn{3}{l}{ Mean absolute error $(\mathrm{sec})$} & \multicolumn{3}{c}{ Mean percentage error (\%) } & \multicolumn{3}{c}{ Interval relative error (\%) } \\
\cline { 2 - 9 } & SF-type & LI-type & MLI-type & SF-type & LI-type & MLI-type & SF-type & LI-type & MLI-type \\
\hline$\delta=1 \mathrm{sec}$ & 0.0316 & 0.0004 & 0.0003 & 0.0155 & 0.0002 & 0.0002 & 3.1619 & 0.0358 & 0.0331 \\
$\delta=5 \mathrm{sec}$ & 0.1552 & 0.0084 & 0.0067 & 0.0766 & 0.0040 & 0.0030 & 3.1031 & 0.1687 & 0.1332 \\
$\delta=10 \mathrm{sec}$ & 0.3157 & 0.0297 & 0.0195 & 0.1563 & 0.0143 & 0.0089 & 3.1570 & 0.2969 & 0.1952 \\
$\delta=20 \mathrm{sec}$ & 0.6157 & 0.1241 & 0.0901 & 0.3009 & 0.0591 & 0.0410 & 3.0787 & 0.6203 & 0.4503 \\
$\delta=30 \mathrm{sec}$ & 1.0066 & 0.1647 & 0.1059 & 0.4966 & 0.0792 & 0.0481 & 3.3553 & 0.5491 & 0.3531 \\
$\delta=40 \mathrm{sec}$ & 1.2636 & 0.4887 & 0.3481 & 0.6389 & 0.2334 & 0.1595 & 3.1590 & 1.2218 & 0.8703 \\
$\delta=60 \mathrm{sec}$ & 1.9487 & 0.3426 & 0.2095 & 0.9860 & 0.1694 & 0.1002 & 3.2478 & 0.5710 & 0.3491 \\
\hline
\end{tabular}

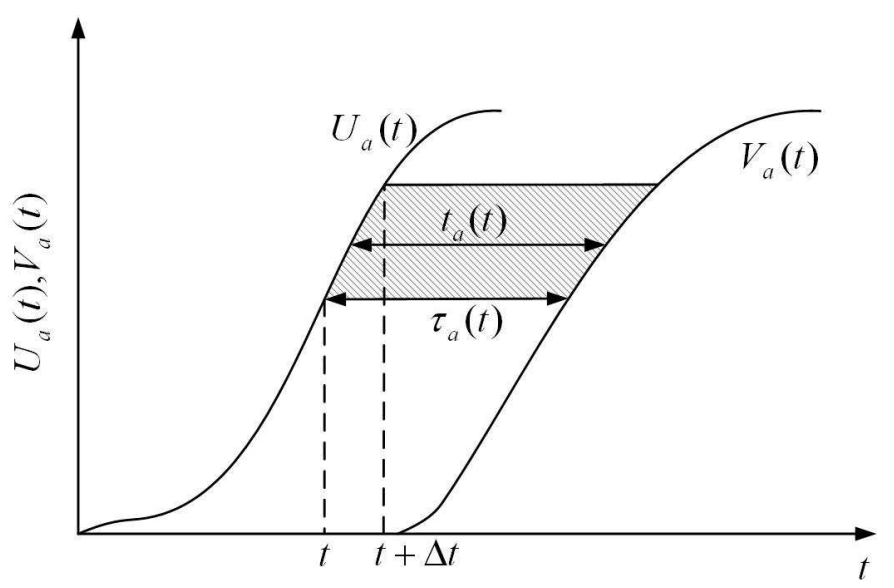

Figure 1: Cumulative vehicle numbers as a function of time.

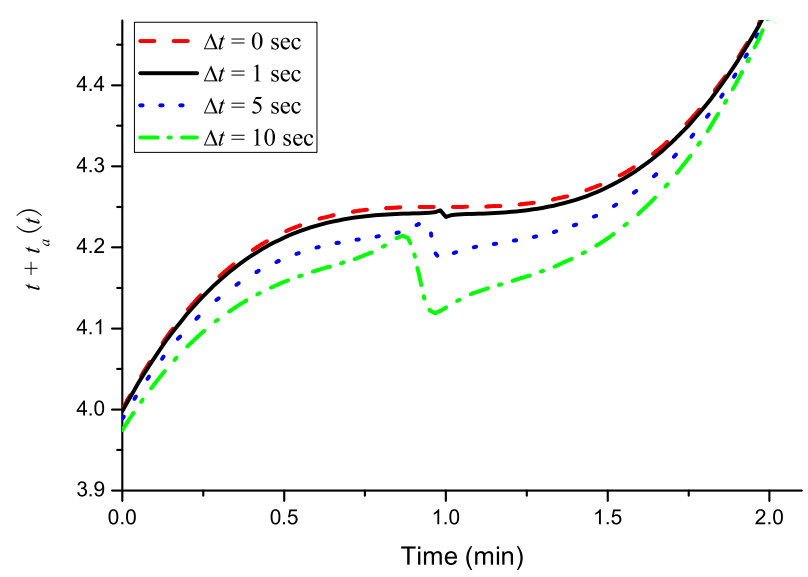

Figure 2: The profiles of $t+t_{a}(t)$ with various interval lengths in Example 1. 


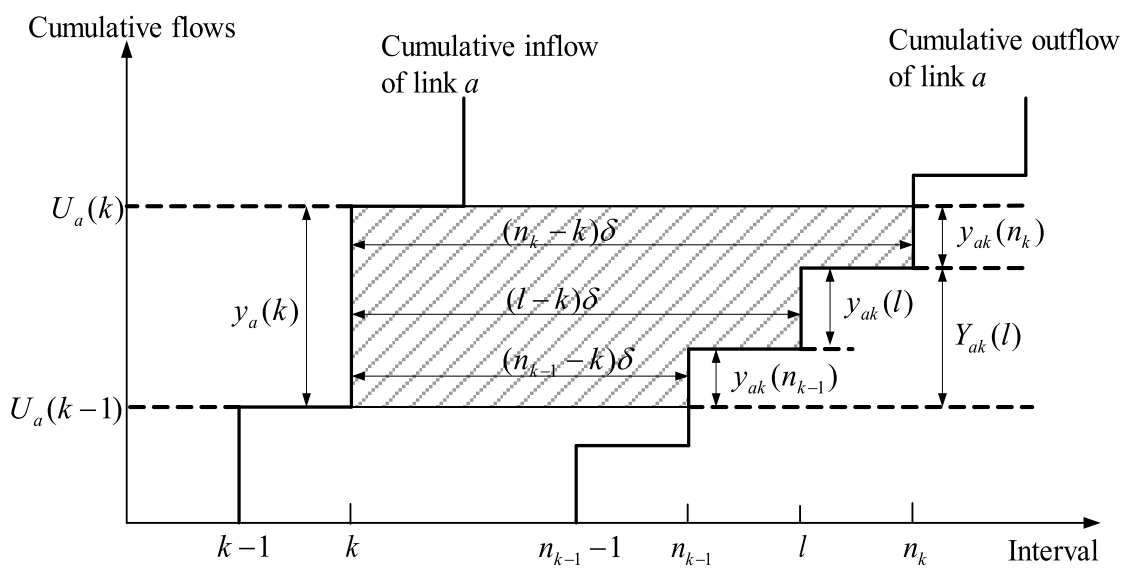

Figure 3: Cumulative flow functions approximated by step functions.

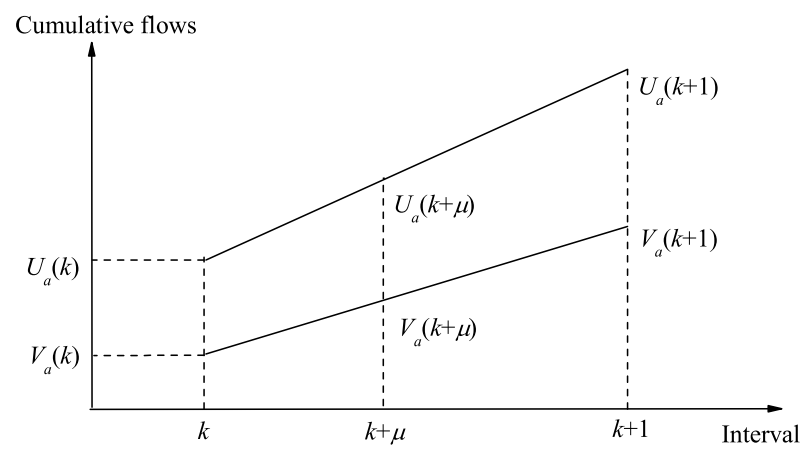

Figure 4: Linear interpolation of cumulative flows. 


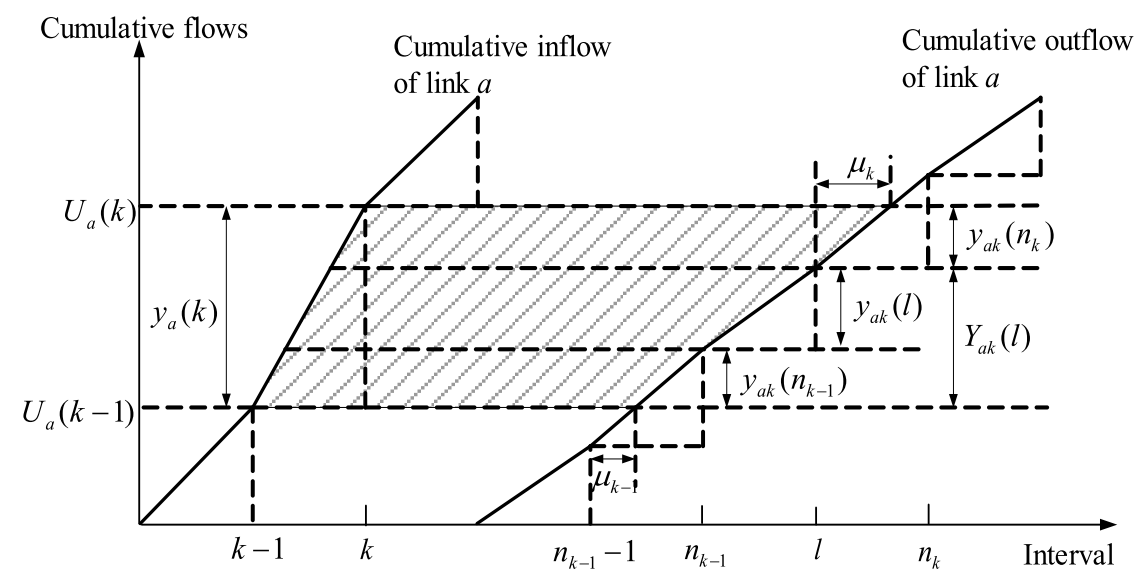

Figure 5: Linear interpolation of cumulative flows of link $a$.

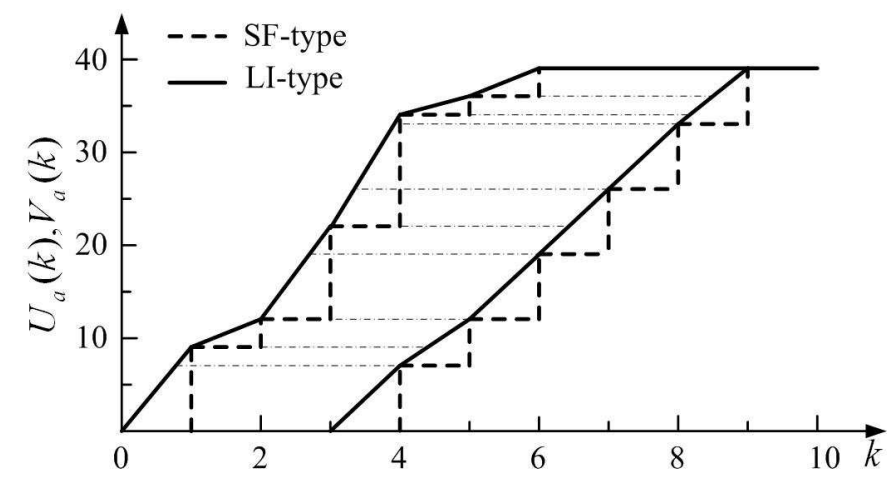

Figure 6: Cumulative flow curves for Example 2.

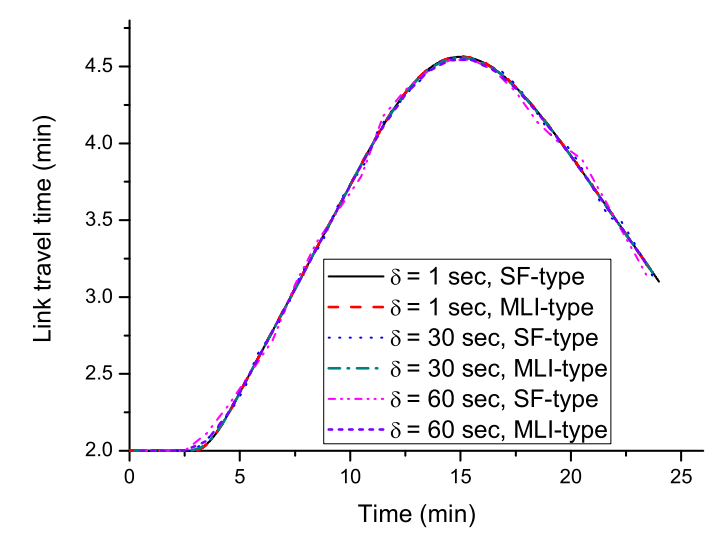

Figure 7: Link travel time profiles for Example 4. 